\title{
Age-related differences in the neural network dynamics underlying the predictability gain
}

\author{
Anna Uta Rysop ${ }^{1}$, Lea-Maria Schmitt ${ }^{2,3}$, Jonas Obleser ${ }^{2,3}$, Gesa Hartwigsen ${ }^{1}$
}

1 Lise Meitner Research Group Cognition and Plasticity, Max Planck Institute for Human Cognitive and Brain Sciences, Stephanstrasse 1a, 04103 Leipzig, Germany

2 Department of Psychology, University of Lübeck, Ratzeburger Allee 160, 23562 Lübeck, Germany

${ }^{3}$ Center of Brain, Behavior and Metabolism, University of Lübeck, Ratzeburger Allee 160, 23562 Lübeck

\section{Correspondence}

Anna Rysop \& Gesa Hartwigsen

Lise Meitner Research Group Cognition and Plasticity

Max Planck Institute for Human Cognitive and Brain Sciences

Stephanstr. 1a, D-04103 Leipzig

Email: rysop@cbs.mpg.de | hartwigsen@cbs.mpg.de 


\section{Abstract}

Speech comprehension is often challenged by increased background noise, but can be facilitated via the semantic context of a sentence. This predictability gain relies on an interplay of language-specific semantic and domain-general brain regions. However, age-related differences in the interactions within and between semantic and domain-general networks remain poorly understood. Here we investigated commonalities and differences in degraded speech processing in healthy young and old participants. Participants performed a sentence repetition task while listening to sentences with high and low predictable endings and varying intelligibility. Stimulus intelligibility was adjusted to individual hearing abilities. Older adults showed an undiminished behavioural predictability gain. Likewise, both groups recruited a similar set of semantic and cingulo-opercular brain regions. However, we observed age-related differences in effective connectivity for high predictable speech of increasing intelligibility. Young adults exhibited stronger coupling within the cingulo-opercular network and between a cingulo-opercular and a posterior temporal semantic node. Moreover, these interactions were excitatory in young adults but inhibitory in old adults. Finally, the degree of the inhibitory influence between cingulo-opercular regions was predictive of the behavioural sensitivity towards changes in intelligibility for high predictable sentences in older adults only. Our results demonstrate that the predictability gain is relatively preserved in older adults when stimulus intelligibility is individually adjusted. While young and old participants recruit similar brain regions, differences manifest in network dynamics. Together, these results suggest that ageing affects the network configuration rather than regional activity during successful speech comprehension under challenging listening conditions.

Keywords: speech in noise, semantic predictability, cingulo-opercular network, effective connectivity, ageing 


\section{Introduction}

In everyday conversations, speech comprehension is often hampered by challenging listening conditions, such as environmental noise. To maintain successful communication, listeners exploit facilitating factors, such as semantic context. The semantic context of a sentence provides information that can be used to predict upcoming words ("predictability gain"). This predictability gain has its maximum impact on comprehension at intermediate levels of intelligibility, that is, when the acoustic speech stream is neither completely intelligible nor completely unintelligible (Hartwigsen, Golombek, \& Obleser, 2015).

With respect to the neural correlates underlying the predictability gain, several neuroimaging studies found consistent activation in left (and less consistently right) angular gyrus (AG), left posterior middle temporal gyrus (pMTG) and left inferior frontal gyrus (IFG; Adank, 2012; Golestani, Hervais-Adelman, Obleser, \& Scott, 2013; Obleser \& Kotz, 2010; Obleser, Wise, Dresner, \& Scott, 2007; Rysop, Schmitt, Obleser, \& Hartwigsen, 2021). These regions have been suggested to constitute key regions of semantic processing (Binder, Desai, Graves, \& Conant, 2009; Jefferies, 2013; Seghier, 2013). In addition, some studies also showed a tighter coupling of left angular gyrus with other nodes of the semantic network when listening to high versus low predictable sentences at intermediate levels of intelligibility (Obleser et al. 2007; Obleser and Kotz, 2010; Golestani et al. 2013). Other work demonstrated the functional relevance of left angular gyrus for the predictability gain with inhibitory neurostimulation (Hartwigsen et al. 2015). Together, these studies provide evidence for a key role of semantic regions, particularly left angular gyrus, in the successful integration of semantic information into sentential context under challenging listening conditions in healthy young adults.

Aside from the contribution of semantic regions, sentence comprehension under challenging listening conditions results in increased executive demands (Desjardins \& Doherty, 2013; Fitzhugh, Schaefer, Baxter, \& Rogalsky, 2021), leading to a recruitment of domaingeneral networks. Specifically, the cingulo-opercular network (Dosenbach, Fair, Cohen, Schlaggar, \& Petersen, 2008; Uddin, Yeo, \& Spreng, 2019), encompassing bilateral anterior insulae as well as a portion of the dorsal anterior cingulate and pre-supplementary motor area (pre-SMA), is the most frequently reported network in degraded speech processing (Eckert, Teubner-Rhodes, \& Vaden Jr., 2016; Erb \& Obleser, 2013; Vaden Jr., Kuchinsky, Ahlstrom, Dubno, \& Eckert, 2015; Vaden Jr. et al., 2013; Vaden Jr., Teubner-Rhodes, Ahlstrom, Dubno, \& Eckert, 2017, Rogers \& Peelle, 2021). Prior studies investigated the contribution of cinguloopercular regions to challenging listening conditions either by using manipulations of the auditory signal itself (e.g., noise vocoding, where spectral information of an acoustic signal is 
degraded while temporal information is preserved, see Shannon, Zeng, Kamath, Wygonski, \& Ekelid, 1995) or by masking the acoustic signal with broadband noise or multi-talker babble (Darwin, 2008; Miller, 1947). Although increased activity in cingulo-opercular regions has been linked to error monitoring, executive functions and maintenance of auditory attention (Wilsch, Henry, Herrmann, Maess, \& Obleser, 2015), its exact role in acoustically-demanding speech perception remains vaguely defined.

In one study, increased activity in cingulo-opercular regions during challenging listening conditions was predictive of successful word recognition in the following trial (Vaden et al. 2013), thereby indicating that the functional dynamics within this network are directly relevant to behaviour. In line with this observation, Rysop et al. (2021) demonstrated that stronger inhibition between nodes of the cingulo-opercular network at intermediate levels of intelligibility was associated with an increased predictability gain. These findings suggest that active inhibition of the cingulo-opercular network is beneficial in young adults, as long as semantic cues can be efficiently used to support comprehension of the distorted signal. Otherwise, increased activity in cingulo-opercular regions might help to maintain task performance. Yet, the contribution of the cingulo-opercular network to challenging listening conditions in the ageing brain is unclear.

Ageing is associated with a decline across sensory and cognitive domains such as hearing acuity, processing speed or working memory capacity (Salthouse, Atkinson, \& Berish, 2003; Wingfield, Amichetti, \& Lash, 2015), while semantic abilities are thought to remain relatively stable across the lifespan (Verhaeghen, 2003; Wingfield \& Stine-Morrow, 2000). While listening to degraded speech is already demanding for young listeners, older adults frequently report markedly enhanced difficulties in understanding speech in challenging listening situations, even if they show no signs of age-related hearing loss or difficulties in ideal listening conditions (Gordon-Salant, 2005; Gordon-Salant \& Fitzgibbons, 1995; Humes, 1996; PichoraFuller, Alain, \& Schneider, 2017). With regard to the predictability gain, behavioural studies point towards either an increased reliance (Goy, Pelletier, Coletta, \& Pichora-Fuller, 2013; Pichora-Fuller, 2008; Pichora-Fuller, Schneider, \& Daneman, 1995) or similar use of contextual information in older compared to younger adults (Dubno, Ahlstrom, \& Horwitz, 2000; Sheldon, Pichora-Fuller, \& Schneider, 2008; MacDonald et al., 2008). For example, in a semantic priming paradigm with an acoustically degraded prime, older participants benefitted from contextual information as much as young participants (Sheldon et al. 2008).

While the beneficial effect of semantic context generally seems to be preserved in older adulthood, changes at the neural level remain debated. Some authors argue that the reduced magnitude of electrophysiological measures to high predictable sentences in old versus young adults might reflect less efficient use of sentential context (Federmeier, 2007; Payne \& 
Federmeier, 2018; Wlotko, Federmeier, \& Kutas, 2012). Likewise, a recent meta-analysis showed less activation in semantic key regions for older participants but increased recruitment of domain-general regions during semantic tasks (Hoffman \& Morcom, 2018). Yet, these changes were mainly observed when task performance was poor. Thus, it remains unclear whether increased recruitment of domain-general networks in the ageing brain reflects a (compensatory) attempt to maintain performance during speech comprehension under challenging listening conditions (Eckert et al., 2008; Peelle, 2012; Peelle, Troiani, Grossman, \& Wingfield, 2011) or rather a pattern of dedifferentiation, indicating less functional specialization (Li, Lindenberger, \& Sikström, 2001). Some authors suggest that older adults already recruit such domain-general resources under relatively easy conditions (Erb \& Obleser, 2013; Fitzhugh, Braden, Sabbagh, Rogalsky, \& Baxter, 2019; Peelle, 2018). Accordingly, stronger activity in domain-general regions was associated with better comprehension of degraded auditory sentences selectively in old adults (Erb \& Obleser, 2013). These findings suggest that the (potentially compensatory) upregulation of activity in domaingeneral areas is more pronounced in older listeners.

The additional upregulation of domain-general regions is accompanied by altered patterns of functional connectivity. It has been consistently reported that older adults exhibit decreased levels of connectivity within domain-general networks including the cingulo-opercular network (Geerligs et al., 2015), while connectivity between networks was increased at rest (Zonneveld et al. 2019, Setton et al. 2021) and during cognitive tasks (Zhang et al. 2021). However, studies addressing age-differences in task-related connectivity during language processing are sparse. Hence, it remains unclear how ageing affects functional interactions within and between networks during speech comprehension under adverse listening conditions.

In the present study, we explored age-related differences in task-related activity and effective connectivity while participants listened to sentences of varying acoustic intelligibility and semantic predictability. In contrast to previous studies, we used an optimized adaptive tracking paradigm that considered individual differences in auditory perception by adjusting stimulus intelligibility to individual hearing abilities. This assures comparable task performance across participants, which is particularly important in light of variable hearing performance in old participants.

We expected a beneficial effect of semantic context when speech is acoustically degraded in both age groups, as intelligibility levels were adjusted to individual hearing abilities. At the neural level, we expected potentially weaker engagement of semantic regions and relatively stronger recruitment of cingulo-opercular regions in older listeners. Age-related differences should also be reflected in decreased connectivity within the cingulo-opercular and semantic 
network and increased connectivity between the semantic and cingulo-opercular network in older listeners, reflecting increased recruitment of domain-general resources.

To foreshadow our results, when controlling for speech-to-noise reception thresholds as done in the present study, no age-related differences in the behavioural performance or at the level of neural activation patterns were observed. However, relative to young listeners, older listeners showed decreased effective connectivity within the cingulo-opercular network and between left anterior insula and posterior middle temporal gyrus when predictability was high and intelligibility increased. Stronger inhibitory influence between cingulo-opercular regions was associated with better task performance selectively in older listeners, demonstrating its behavioural relevance. 


\section{Materials and Methods}

\section{Participants}

Thirty healthy middle-aged to old German native speakers were recruited for the fMRI experiment. Inclusion criteria comprised age-normal hearing (tested with pure-tone audiometry; see below for further details) and right-handedness according to the German version of the Edinburgh Handedness Inventory (Oldfield, 1971). Participants were excluded if they showed signs of cognitive impairment (Mini Mental State Examination score $<27$; MMSE; Folstein, Folstein, \& McHugh, 1975) or reported a history of neurological or psychiatric disorders. Data from three participants were excluded from the analyses due to excessive head movement during fMRI (movement parameter exceeded 1.5 times the voxel size), and data from one participant because of a ceiling effect in the behavioural performance.

The final sample size was $n=26$ ( $M=62$ years, range: $50-77$ years, 19 female, see Supplementary Table 1 for further information). This group was compared to 26 healthy young participants ( $M=25$ years, range: 19-29 years, 15 female), who had performed the same task under exact same procedures (see (Rysop et al., 2021) for a detailed analysis of the neural predictability gain in this younger group). Participants were reimbursed and gave written informed consent in accordance to the declaration of Helsinki prior to participation. The study was approved by the local ethics committee (University of Leipzig).

\section{Experimental Procedure}

The experiment consisted of two sessions. In the first session, each participant's hearing status and cognitive abilities were tested using pure-tone audiometry and MMSE. Only participants with relatively well-preserved peripheral hearing abilities (pure-tone average $<25 \mathrm{~dB} H \mathrm{HL}$ in the listener's better ear) were included. The hearing test was conducted in a soundproof chamber using an audiometer (Oscilla SM910-B Screening Audiometer). Pure-tone averages were computed across frequencies from $250 \mathrm{kHz}$ to $8000 \mathrm{kHz}$ in the right and left ear separately (see Figure 1A for an illustration of the peripheral hearing status of included participants). For included participants, the first session proceeded with further neuropsychological assessments. Working memory was tested using the auditory forward and backward version of the Digit Span Task (Wechsler, 1955). Measures of executive functions (processing speed and cognitive flexibility) were assessed with both versions of the Trail Making Test (Reitan, 1958). Further, we tested verbal fluency and flexibility using two subtests of a German word fluency test (Regensburger Wortflüssigkeitstest; Aschenbrenner, Tucha, \& Lange, 2000). In this test, participants had to generate as many nouns as possible either for one specific semantic category (subtest A, category food) or two alternating semantic categories (subtest 
B, categories fruits and sports) within two minutes. The first session had a duration of approximately one hour.

The fMRI experiment was conducted in a second session on a separate day. Younger participants only took part in this second session. Participants were placed in the scanner and equipped with MR compatible in-ear headphones (MR Confon; Magdeburg, Germany). The volume was initially set to a comfortable intensity. To determine the participants' in-scanner speech reception threshold (SRT; i.e., the signal-to-noise ratio (SNR) required to correctly repeat a sentence with a probability of $50 \%$ ), we conducted an adaptive up-down staircase experiment (Kollmeier et al. 1988; for a detailed description of the procedure see Rysop et al., 2021). The SRT was used as a reference to adjust stimulus intelligibility for each subject individually in the main experiment, thereby correcting for differences in peripheral hearing and ensuring comparable task performance across subjects.

In the main experiment, participants listened to sentences while viewing a white fixation cross on a black screen. Participants were instructed to overtly repeat the sentence as accurately as possible as soon as a green traffic light appeared on the screen (see Figure 1B for an overview of the experimental design). If they had not understood anything, they were asked to say so. Overt responses were recorded for a period of 5 seconds using a FOMRI-III microphone (Optoacoustics, Yehuda, Israel). The intertrial interval varied between 2000 and $7000 \mathrm{~ms}$ and served as an implicit baseline for the fMRI analysis. Stimuli were presented in six blocks using Presentation (version 18.0, Neurobehavioral Systems, Berkeley, USA, www.neurobs.com). The fMRI experiment had a duration of 50 minutes, the total duration of the second session was approximately 90 minutes. 
A

Audiograms of older listeners

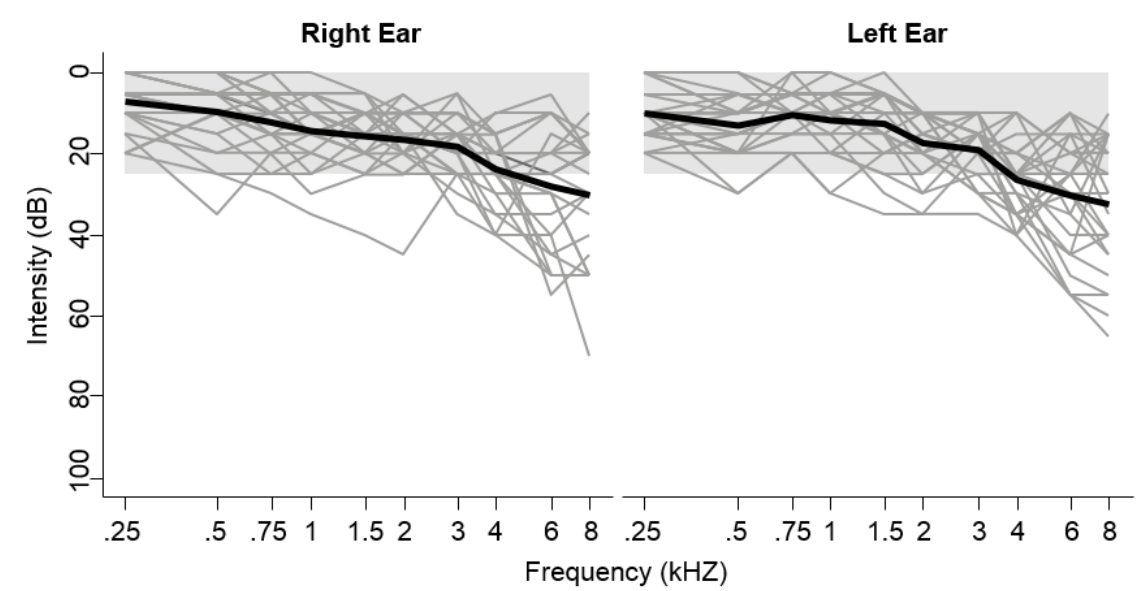

B

Task Design
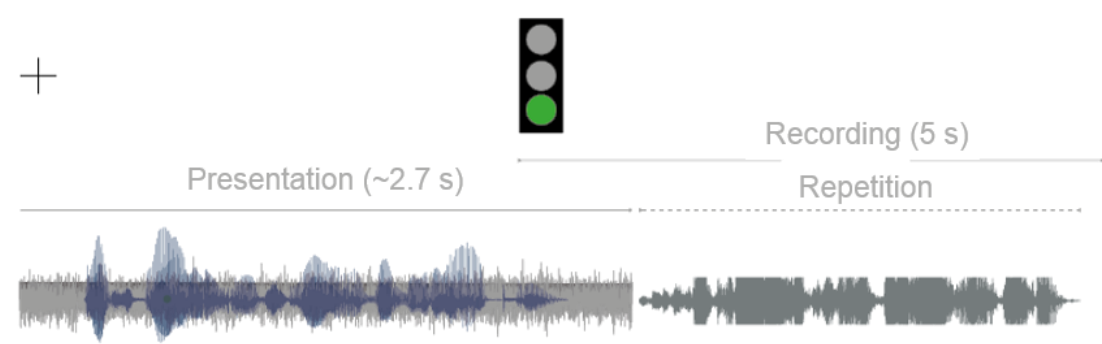

High Predictability

Intelligibility $(\mathrm{dB})$

Sie kürzte den Saum ihres neuen

She shortened the hem of her new

Low Predictability

Sie musste nach Hause wegen ihres

She had to go home because of her

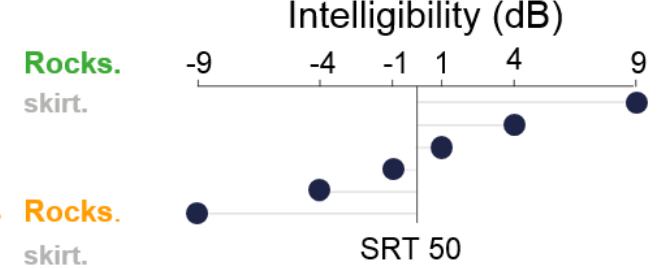

Figure 1. Experimental procedures A. Pure-tone audiograms for the older participants, plotted for the right and left ear separately. Grey lines represent the hearing curve of single participants across each tested frequency; the bold black line represents the average across participants. The intensity range for normal hearing is shaded in grey. B. In the sentence repetition task, each trial consisted of a listening period in which a short sentence (blue waveform) was embedded in speech-shaped noise (grey waveform) and a fixation cross was presented on the screen. With the onset of the sentence-final word (i.e., keyword), a green traffic light appeared and marked the recording period, in which participants were asked to repeat the sentence as accurately as possible. The sentence context was either highly (green) or lowly (orange) predictive of the sentence-final keyword. The signal-to-noise ratio of the sentences against the speech-shaped background noise was adjusted to individual speech reception thresholds (SRT 50).

\section{Stimulus Material}

Experimental sentences were taken from the German Speech Intelligibility in Noise (G-SPIN) material (Kalikow, Stevens, \& Elliott, 1977; for a detailed description of the German version, see Erb, Henry, Eisner, \& Obleser, 2012). The 216 sentences consisted of pairs with the same sentence-final words (i.e., keyword) but different preceding sentence frames: While the frame of one of the sentences was predictive of the keyword (high predictability: "She shortened the hem of her new skirt."), the other one was not (low predictability: "She had to go home because of her skirt'). Twenty additional G-SPIN sentences with highly predictable keywords were used for the adaptive tracking procedure. 
The intelligibility of the sentences was manipulated by varying the sentence intensity relative to constant speech-shaped noise in the background. Sentence intensity was varied symmetrically and relative to the previously determined SRT in six steps $(-9 \mathrm{~dB},-4 \mathrm{~dB},-1 \mathrm{~dB}$, $+1 \mathrm{~dB},+4 \mathrm{~dB},+9 \mathrm{~dB}$ SNR relative to the individual SRT; Rysop \& Schmitt et al. 2021 for details). These intelligibility levels were chosen to cover the full range of behavioural performance in each participant while sampling those (intermediate) intelligibility levels with the largest predictability gain more densely.

Experimental conditions yielded a factorial design with the factors predictability (high, low) and intelligibility ( $-9 \mathrm{~dB},-4 \mathrm{~dB},-1 \mathrm{~dB},+1 \mathrm{~dB},+4 \mathrm{~dB},+9 \mathrm{~dB}$ SNR relative to SRT), with 18 sentences per condition. Sentences were presented in a pseudorandomized order with the restriction that each intelligibility level was not presented more than three times in a row to prevent adaptation.

\section{MRI acquisition}

Functional MRI data were collected on a 3 Tesla Siemens Prisma Scanner with a 32-channel head coil. We used a dual gradient-echo planar imaging multiband sequence (Feinberg et al., 2010). The scanning parameters were: $T R=2,000 \mathrm{~ms}$; $\mathrm{TE}=12 \mathrm{~ms}$, $33 \mathrm{~ms}$; flip angle $=90^{\circ}$; voxel size $=2.5 \times 2.5 \times 2.5 \mathrm{~mm}$ with an interslice gap of $0.25 \mathrm{~mm}$; FOV = $204 \mathrm{~mm}$; multiband acceleration factor $=2$. For each participant, 1,500 volumes à 60 slices were acquired in axial direction and interleaved order. Slices were tilted by $10^{\circ}$ off the AC-PC line to increase coverage of anterior temporal lobe regions. Field maps were acquired for later distortion correction (TR $=620 \mathrm{~ms}$; TE $=4 \mathrm{~ms}, 6.46 \mathrm{~ms}$ ). Additionally, high-resolution T1-weighted images were either obtained from the in-house database if available or were acquired in the second session using an MPRAGE sequence (whole brain coverage, TR $=1300 \mathrm{~ms}$, TE $=$ $2.98 \mathrm{~ms}$, voxel size $=1 \times 1 \times 1 \mathrm{~mm}$, matrix size $=256 \times 240 \mathrm{~mm}$, flip angle $\left.=9^{\circ}\right)$.

\section{Data Analysis}

\section{Behavioural Analyses}

All participants' spoken response recordings were cleaned from scanner noise using Audacity (version 2.2.2, https://www.audacityteam.org/) and transcribed by one rater, who additionally determined speech onset times and speech durations. Repetitions of the keywords were rated either as correct or incorrect before calculating the proportion of correctly repeated keywords per participant and condition. Thereafter, psychometric curves were fitted to keyword repetition accuracies across intelligibility levels, separately for sentences with low and high predictability. More specifically, we employed cumulative Gaussian sigmoid functions using the Psignifit toolbox (Fründ, Haenel, \& Wichmann, 2011) in MATLAB (version R2018b, MathWorks; see Supplementary Material for further details). 
All empirical deviances between the fitted and a saturated model fell within the $97.5 \%$ confidence interval of their respective reference distribution, indicating that psychometric curves properly represented the observed behavioural data in every participant. There was no significant difference between the deviance of fits for sentences with low vs. high predictability $\left(\mathrm{t}_{24}=0.11, \mathrm{p}=.916, \mathrm{r}=0.06, \mathrm{BF} 10=0.21\right)$. To illustrate goodness of fit, we additionally calculated the $\mathrm{R}^{2} \mathrm{KL}$, which is based on the information-theoretic measure of Kullback-Leibler divergence and represents the reduction of uncertainty by the fitted model relative to a constant model (Cameron \& Windmeijer, 1997). On average, psychometric curves of sentences with high predictability yielded an $\mathrm{R}^{2} \mathrm{KL}$ of 0.96 ( $\mathrm{SD}=0.04$, range: $\left.0.86-0.995\right)$, sentences with low predictability an $R^{2}{ }_{K L}$ of $0.94(S D=0.05$, range: $0.81-0.996)$.

To quantify the predictability gain, we compared threshold and slope parameters of the psychometric functions for sentences with low versus high predictability. The threshold parameter represents the intelligibility level at which participants correctly repeat half of the keywords. A smaller threshold parameter for sentences with high compared to low predictability would indicate a stronger benefit from semantic context already at levels of lower intelligibility. The slope parameter describes the steepness of the curve at a proportion correct of 0.5 and denotes the sensitivity to changes in intelligibility at intermediate levels. Parameter estimates of the two psychometric curves were analysed using an ANOVA with the factors age and predictability. Furthermore, we analysed speech onset times (SOTs) as a measure of response speed. SOTs were log-transformed and submitted to a three-way ANOVA with the factors age, predictability and intelligibility. Statistical analyses were performed with RStudio (version 4.0.2; R Core Team, 2018) and JASP (version 0.9.1; https://jasp-stats.org/; Wagenmakers et al., 2018).

\section{Functional MRI analyses}

Functional MRI data were preprocessed and analysed in SPM12 (version 7219, Wellcome Department of Imaging Neuroscience, London, UK) and MATLAB (version R2018b). As a first step, the images of the two echo times were combined based on a weighted average of the voxel-wise temporal signal-to-noise ratio maps of the first 30 volumes and realigned using a custom Matlab script. The rationale behind this approach is to improve signal quality in brain regions that typically suffer from signal loss (e.g., anterior temporal lobes; see Halai, Welbourne, Embleton, \& Parkes, 2014 for a similar approach). Further, fMRI data were distortion corrected, co-registered to individual high-resolution structural T1-scans and spatially normalized to the standard template by the Montreal Neurological Institute (MNI). We preserved the original voxel size and finally smoothed the data, using a $5 \mathrm{~mm}^{3} \mathrm{FWHM}$ Gaussian kernel to allow statistical inference based on Gaussian random-field theory (Friston, Worsley, Frackowiak, Mazziotta, \& Evans, 1994). 
Preprocessed data were submitted to a general linear model (GLM). Sentences presentation times were modelled with stick functions and convolved with SPM's canonical hemodynamic response function. We included one regressor of no interest capturing the verbal responses using participant- and trial-specific speech onset times and durations. As additional nuisance regressors we included the six motion parameters obtained from the rigid-body transformation of the realignment step (see Supplementary Figure 1 and Supplementary Table 2) and a vector for each volume that exceeded a framewise displacement of $0.9 \mathrm{~mm}$ (Siegel et al., 2014). A high-pass filter with a cut-off at $128 \mathrm{~s}$ was applied to the data.

At the single-participant level, we computed direct contrasts between experimental conditions. These contrasts encompassed the main effect of intelligibility (i.e., linear increase in intelligibility, $-9<-4<-1<+1<+4<+9 \mathrm{~dB}$ SNR), the main effect of predictability (high predictable > low predictable sentences and vice versa) and the interaction between predictability and intelligibility (high predictable > low predictable sentences, with a linear increase in intelligibility and vice versa). The first-level interaction contrasts were further used to extract the timeseries for the effective connectivity analysis.

The resulting contrast maps were submitted to one-sample t-tests, to investigate effects within each age group. Two-sample t-tests were used to investigate differences between groups. To characterize commonly activated regions by both groups, the respective contrast maps were submitted to a conjunction analysis based on the minimum statistic (Nichols, Brett, Andersson, Wager, \& Poline, 2005). All maps were thresholded at a cluster-level threshold of $p<0.05$ (corrected for family wise error (FWE)) with a voxel-wise threshold of $p<0.001$ (uncorrected) and visualized with BrainNet Viewer (Xia, Wang, \& He, 2013). Anatomical locations were specified using the SPM anatomy toolbox (version 3.0; Eickhoff et al., 2007, 2005) and the Harvard-Oxford atlas (https://fsl.fmrib.ox.ac.uk/fsl/fslwiki/).

\section{Dynamic Causal Modelling (DCM)}

We used Dynamic Causal Modelling (DCM; version 12.5; Friston, Harrison, \& Penny, 2003) and Parametric Empirical Bayes (PEB; Zeidman, Jafarian, Corbin, et al., 2019; Zeidman, Jafarian, Seghier, et al., 2019) to investigate age-related differences in effective connectivity between semantic and domain-general regions. DCM is a method that uses a biophysically informed generative model to estimate effective connectivity between a set of brain regions. The estimated signal is compared to the measured fMRI BOLD signal and quantified in terms of negative variational free energy. Three sets of parameters have to be defined: (1) intrinsic parameters, reflecting endogenous connectivity between regions in the absence of experimental manipulation (A-Parameters), (2) modulatory parameters that encode the rates of change in connectivity due to an experimental manipulation (B-Parameters) and (3) driving 
input parameters, reflecting the influence of an experimental task on single nodes within the network (C-Parameters).

While the classical DCM approach allows to investigate the network architecture (or configuration) underlying an experimental effect, PEB spans a hierarchical model over the connectivity parameters, allowing to quantify differences and commonalities in coupling strengths among groups of participants (Friston et al., 2016; Zeidman, Jafarian, Corbin, et al., 2019). PEB provides a Bayesian GLM-like approach that divides inter-subject variability into regressor effects and unexplained random effects (Zeidman, Jafarian, Seghier, et al., 2019). In contrast to classical inference over parameter estimates, the PEB framework does not only take the mean but also the uncertainty of individual parameters into account. Consequently, participants with more uncertain parameters will influence the group estimates less than participants with more certain parameters (Zeidman, Jafarian, Seghier, et al., 2019).

We were interested in the context-dependent effective connectivity within the nodes of the semantic and cingulo-opercular network as well as between both networks. We approached these questions in a two-step procedure. First, we performed a standard DCM analysis within the cingulo-opercular network, motivated by the findings of our previous study, to test the goodness of fit of the winning model that was identified in young participants (Rysop et al., 2021). In brief, we found that the data of young participants was best explained by a model in which intelligibility and predictability of the sentences jointly modulated the connections from pre-SMA and right anterior insula to the left anterior insula. This model had a relatively high exceedance probability of 0.73 . Here, we aimed at testing whether the optimal model for young participants also succeeds in explaining the older participants' data.

Second, we employed the DCM PEB approach which enabled us to test for age-related effects in a larger model space encompassing nodes of the semantic and cingulo-opercular network identified by the conjunction analysis. Specifically, we asked how predictability and intelligibility of sentences affects the coupling (1) within the semantic and cingulo-opercular network, (2) between both networks, as well as (3) whether coupling strengths differ across groups and (4) whether age-related differences in coupling strength are related to behavioural differences.

\section{Seed Region Selection}

For the standard DCM approach, we defined the seed regions of the cingulo-opercular network based on the interaction contrast in the older subgroup. The seed regions comprised the preSMA [-4 20 42] and anterior insulae [left: -30 23 -2, right: 3326 -2]. For the PEB DCM approach, we defined the seed regions based on the results of the conjunction analyses and on our previous findings in young participants (Rysop et al., 2021). Seed regions comprised bilateral 
AG [left: $-42-7035$; right: $46-6435]$ and left pMTG [-57 -60 -2] of the semantic network, as well as pre-SMA [ $\left.\begin{array}{lll}-7 & 13 & 48\end{array}\right]$ and anterior insulae [left: $-3323-2$, right: 3323 0] of the cinguloopercular network. The first principal component (eigenvariate) was extracted from all voxels within a spherical region of interest $\left(6 \mathrm{~mm}\right.$ radius) that passed a liberal threshold of $p_{\text {uncorrected }}$ $<.05$ for each seed region. The sphere was initially centred on the group coordinate and was allowed to move within a spherical search space of $10 \mathrm{~mm}$ around the group coordinate to account for variation in the exact participant-specific maximum. The search space for the bilateral insulae was constrained by a $5 \mathrm{~mm}$ sphere centred on the group coordinate to ensure that the individual peak fell within the anterior insula and not the surrounding regions. The extracted time courses were adjusted by an F-contrast spanning across the experimental conditions to regress out effects of no interest (i.e., overt speech or head movement).

\section{Standard DCM Setup}

For the standard DCM we included a design matrix with one regressor containing the onsets of all experimental stimuli as driving input. Modulatory parameters were incorporated as six regressors encoding high and low predictability at paired intelligibility levels of low ( -9 and -4 $\mathrm{dB}$ SNR relative to SRT), medium ( -1 and $+1 \mathrm{~dB}$ SNR relative to SRT) and high intelligibility (+4 and +9 dB SNR relative to SRT; see Rysop et al., 2021 for a detailed description of this DCM setup).

At the first-level, we specified 84 models per participant. All models had full bidirectional intrinsic connections and inhibitory self-connections. The models differed in the configuration of the driving input (7 variants) and the modulatory input (12 variants). The driving input was set to either one, two or all regions at a time. The modulatory influence was set to either one connection or two afferent or efferent connections at a time. To reduce the model space to a reasonable number, models were grouped in 12 families of models defined by the modulatory input (Penny et al., 2010). Within each family of models the modulatory input was kept constant while the driving input was varied.

At the second-level we identified the most probable family of models given the data using the random-effects Bayesian Model Selection procedure (Penny et al., 2010; Stephan, Penny, Daunizeau, Moran, \& Friston, 2009). In this procedure, each family of models is assigned an exceedance probability that allows to quantify how good the model describes the underlying data. We focussed on the question whether the winning family of models, that we identified in Rysop et al., 2021, would also succeed in explaining the data of the older subgroup. We further extracted the percentage of explained variance to obtain a parameter of the model fit for older participants. Finally, we extracted single-participant modulatory parameter estimates from the winning model of the previous publication (Rysop et al. 2021). Parameter estimates were 
submitted to an independent-samples t-test to investigate age-related differences on modulated connections between regions. Independent-samples t-tests were calculated in JASP (version 0.9.1), parameter estimates were visualized with $R$ (version 4.0.2).

\section{PEB DCM Setup}

The participant-level design matrix for the PEB DCM analysis consisted of three regressors. The first regressor coded the onsets of all experimental stimuli and served as driving input regressor. The ensuing regressors served as modulatory input and coded the effect of 1) high and 2) low predictability with the level of intelligibility added as parametric modulation (i.e., the effect of predictability, scaled linearly with intelligibility).

At the first-level, we specified and inverted one "full" DCM for each participant (see Figure $6 \mathrm{~A}$ for a schematic full model). This model included all possible reciprocal connections between seed regions as well as self-connections. Because we did not include a primary sensory region in the model, we set the driving input to every seed region (C-Matrix). Each connection, except for the self-connections, could receive modulation by experimental inputs (B-Matrix). The inputs were not mean-centered, so that the intrinsic parameter estimates (Amatrix) can be interpreted as baseline connectivity in the absence of a task (Dijkstra, Zeidman, Ondobaka, Van Gerven, \& Friston, 2017; Marreiros, Kiebel, \& Friston, 2008). All full models were inverted using variational Laplace. We extracted the percent variance explained to obtain a parameter for the model fit.

At the second-level, we set up the PEB model of between-subject effects to investigate commonalities and differences in intrinsic and modulatory parameters. The PEB model included a group-level design matrix with one regressor encoding the mean across both groups (or commonalities across participants) whereas the second regressor encoded age group, to identify differences in connectivity between younger and older participants. In contrast to traditional DCM, this procedure does not compare the model evidence of different network architectures, but the effect of the presence or absence of an experimental manipulation on a connection. Thus, each connection, opposed to the whole network architecture, receives a posterior probability. To only keep those parameters with a high probability of contributing to the model evidence, we performed an automatic search over reduced models using Bayesian Model Comparison and Bayesian Model Reduction (BMR; Friston et al., 2016). In this procedure, parameters are systematically switched on and off (i.e., nested models) and compared against the full model, pruning away those parameters that do not contribute to the model evidence (negative variational free energy).

Lastly, we computed a Bayesian Model Average (BMA) over the parameters of the final iteration of the automatic search. Here, parameters were weighted by their posterior 
probabilities (Zeidman, Jafarian, Seghier, et al., 2019). To retain those parameters with 'strong' evidence of being non-zero (Kass \& Raftery, 1995), we applied a threshold at a posterior probability of $>95 \%$ based on free energy. Further, we probed whether the effect sizes were large enough to predict a left-out participant's age group using leave-one-out cross-validation on the estimated parameters that had a significant age-related difference. Finally, to relate differences in connectivity to the behavioural performance, we extracted participant-specific parameters from the connections that showed a significant group effect and correlated these with the respective behavioural parameters of the psychometric curves. 


\section{Results}

\section{Semantic context benefits comprehension under challenging listening conditions independent of age}

The average speech reception threshold obtained during the adaptive tracking procedure was significantly higher for older $(3.75 \mathrm{~dB})$ than for younger participants $(1.68 \mathrm{~dB} ; t(41.12)=2.36$, $p=0.023$; Figure $2 \mathrm{~A}$ ). This finding is in line with a previous report that SRT's are approximately two dB higher in older adults (Schneider, Pichora-Fuller, \& Daneman, 2010).

On average, keyword repetition accuracies were comparable between young ( $\mathrm{M}=52.33$ $\%, S D=8.09 \%$ ) and old participants ( $M=54.59 \%$, SD = $10.56 \%$ ). To explore effects of age on speech comprehension, we fitted psychometric curves to keyword repetition accuracies across intelligibility levels separately for both age groups and predictability levels (Figure 2B). We found a significant main effect of predictability for the threshold parameter of psychometric curves $\left(F(1,50)=302.3, p<0.001, \eta^{2}=0.41\right.$; Figure $\left.2 C\right)$, indicating that high predictability benefits comprehension already at lower levels of intelligibility. Further, a significant main effect of the slope parameters $\left(F(1,50)=7.46, p=0.009, \eta^{2}=0.08\right.$; Figure $\left.2 \mathrm{C}\right)$ indicates that an increase in intelligibility benefits comprehension more strongly when speech is highly predictable. Together these results demonstrate that speech comprehension at intermediate levels of intelligibility generally benefits from high predictability. Most central to the aims of the present study, psychometric curves were strikingly similar between both age groups. Accordingly, the main effect of age and the interaction of age and predictability was not significant for any parameter of the psychometric curves (Supplementary Tables 3 and 4 for all results).

The analysis of log-transformed speech onset times revealed a significant main effect of predictability $(F(1,50)=163.51, p<0.001, \eta=0.038)$, a significant main effect of intelligibility $(F(5,250)=36, p<0.001, \eta=0.08)$ and a significant interaction between predictability and intelligibility $(F(5,250)=23.29, p<0.001, \eta=0.02)$. Post-hoc pairwise comparisons showed that highly predictable sentences were repeated significantly faster than lowly predictable sentences at the three highest intelligibility levels $\left(+1 \mathrm{~dB}\right.$ SNR: $t(101.54)=-2.997, p_{\text {corr }}=0.003$; $+4 \mathrm{~dB}$ SNR: $t(99.3)=-3.57, p_{\text {corr }}<0.001 ;+9 \mathrm{~dB}$ SNR: $\left.t(101.62)=-3.63, p_{\text {corr }}<0.001\right)$. There was no main effect of age $(F(1,50)=0.92, p=0.34, \eta=0.014)$ and no significant interaction of age with intelligibility or predictability (Figure 2D). 
A Speech Reception Threshold

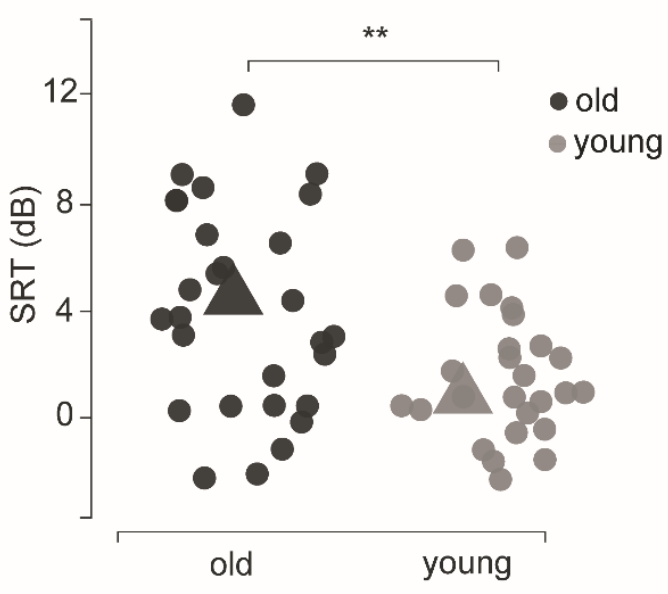

C Threshold

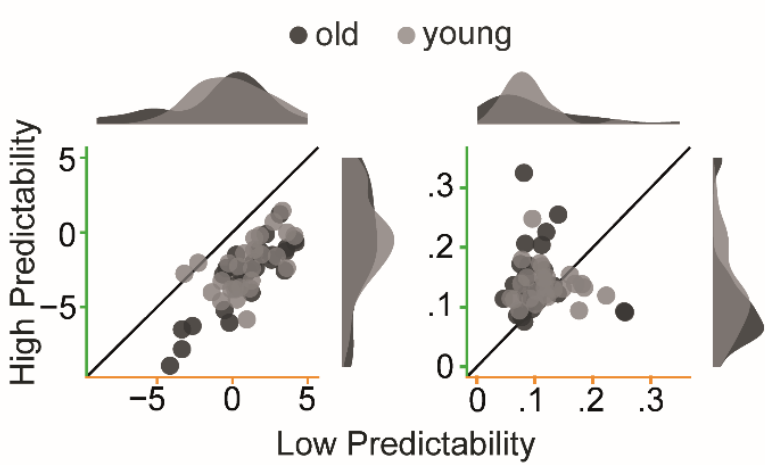

B

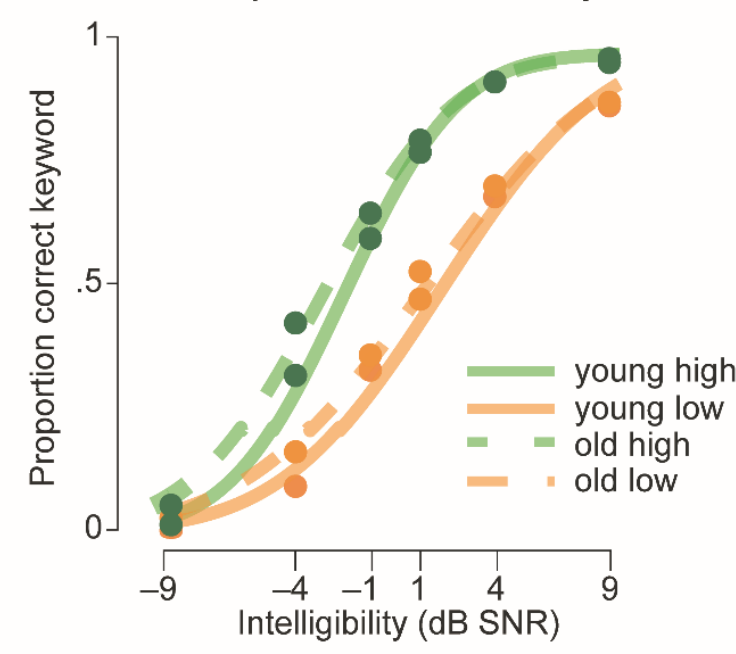

D

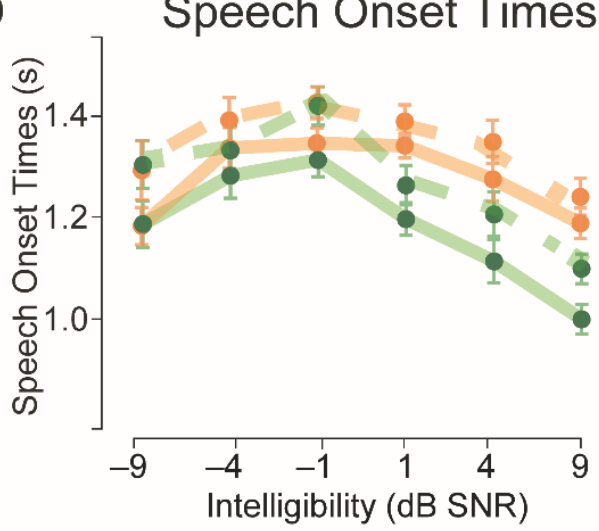

Figure 2. Behavioural results. A Speech reception thresholds (SRT) for old and young participants. Single dots represent individual participants; triangles represent the mean SRT per age group ${ }^{* *}=p<0.01$. B Psychometric curves fitted to the proportion of correctly repeated keywords (dots) across intelligibility levels for high (green) and low (orange) predictable sentences, separately for each age group (solid lines = young, dotted lines = old participants). C Individual threshold (left) and slope (right) parameters extracted from the psychometric curves at $50 \%$ correct for highly (y-axis) and lowly (x-axis) predictable keywords. Dark grey = old participants, light grey = young participants. D. Mean speech onset times for highly (green) and lowly (orange) predictable sentences across intelligibility levels.

\section{Intelligibility effects in bilateral superior temporal cortex are independent of age}

First, we were interested in brain activity sensitive to increasingly intelligible speech, irrespective of its predictability. In the older group, we identified regions encompassing bilateral superior temporal gyrus including primary auditory cortex and bilateral clusters in the somatosensory cortices (pre- and postcentral regions, as well as parietal operculum; Supplementary Figure 2A, Supplementary Table 5). Additionally, subcortical regions such as left putamen and bilateral cerebellum were activated more strongly by more intelligible speech. Speech-sensitive activity in young listeners encompassed large clusters in bilateral superior temporal gyrus, parietal operculum and additional frontal and parietal regions (Supplementary 
bioRxiv preprint doi: https://doi.org/10.1101/2021.12.02.470763; this version posted December 3,2021 . The copyright holder for this preprint (which was not certified by peer review) is the author/funder, who has granted bioRxiv a license to display the preprint in perpetuity. It is made available under aCC-BY-NC-ND 4.0 International license.

Figure 2A, Supplementary Table 5). A conjunction analysis confirmed increased activity in bilateral primary auditory regions in the superior temporal gyri for increasingly intelligible speech comprehension independent of age (Figure $3 \mathrm{~A}$ ).

The direct comparison between age groups revealed increased activation in left and right precentral gyrus as well as left and right cerebellum in older participants. Contrary, older participants exhibited less activation in medial frontal regions than young listeners.

\section{Commonly activated regions by old and young participants}

\section{A Main Effect of Intelligibility}

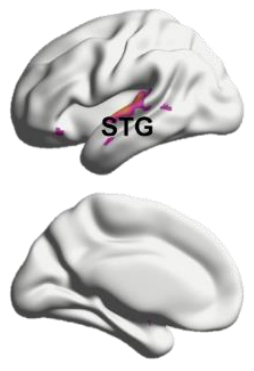

3

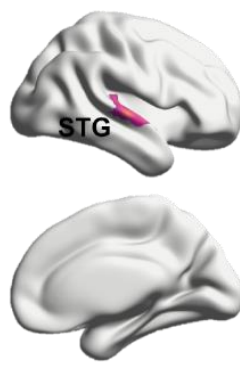

13
B Main Effect of Predictability (high > low)

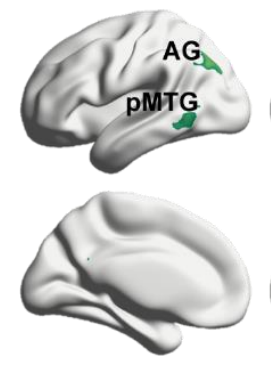

3
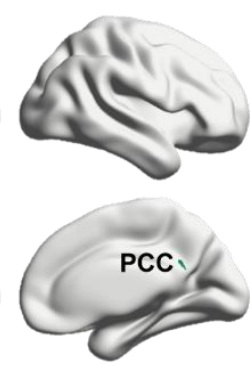

7

T

C Main Effect of Predictability (low $>$ high)

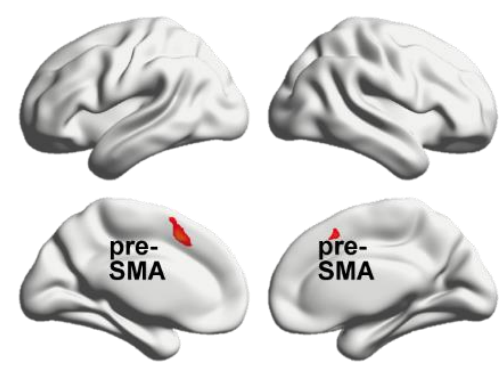

3

Figure 3. Activation maps showing the results of the conjunction analysis. Brain regions displayed are recruited by both age groups. A Brain regions sensitive to increasingly intelligible speech irrespective of predictability, thresholded at peak-level $p_{F W E}<0.05$. B Brain regions sensitive to predictability of speech irrespective of intelligibility for high predictable sentences, thresholded at a voxel-wise $p<0.001$ and a cluster-wise $p_{F W E}<0.05$. C Brain regions sensitive to predictability of speech irrespective of intelligibility for low predictable sentences, thresholded at a voxel-wise $p<0.001$ and a cluster-wise $p_{F W E}<0.05$. STG $=$ superior temporal gyrus, $A G=$ angular gyrus, $\mathrm{pMTG}=$ posterior middle temporal gyrus, $\mathrm{PCC} /$ prec $=$ posterior cingulate cortex/precuneus, pre-SMA = presupplementary motor area.

\section{Semantic regions are commonly recruited when speech is highly predictable}

Next, we were interested in brain regions that support the processing of semantic information, irrespective of intelligibility. Here we identified a set of left-hemispheric and midline regions, encompassing the angular gyrus, posterior middle temporal gyrus, paracingulate gyrus and precuneus that showed stronger activation for high compared to low predictable sentences in old participants (Supplementary Figure 2B, left, Supplementary Table 6). These regions are considered key regions (pMTG and AG) or extended regions (precuneus, posterior cingulate) of the semantic system in young and older adults (Binder et al. 2009; 2011; Hoffman \& Morcom, 2018). Additionally, the cerebellum, bilateral and subcortical subcallosal cortex as well as left-hemispheric hippocampus and amygdala were found to be involved in processing of highly predictable speech in older participants. These regions do not constitute typical core semantic regions, but are sometimes referred to as extended semantic regions (Binder et al. 2009). While cluster sizes were larger for young adults, the activation pattern that emerged was similar (Supplementary Figure 2B). A conjunction analysis showed that activation common 
bioRxiv preprint doi: https://doi.org/10.1101/2021.12.02.470763; this version posted December 3, 2021. The copyright holder for this preprint (which was not certified by peer review) is the author/funder, who has granted bioRxiv a license to display the preprint in perpetuity. It is made available under aCC-BY-NC-ND 4.0 International license.

to both age groups during highly predictable speech was located in left AG, pMTG and precuneus/paracingulate gyrus (Figure 3B). Direct comparisons between age groups revealed increased activation in a large cluster in the precuneus in young compared to older participants during the processing of highly predictable speech. No other differences were found.

\section{Pre-SMA is commonly activated when semantic predictability is low}

Low predictable sentences compared to high predictable sentences elicited stronger activity in the pre-SMA in old participants. Younger adults additionally showed activity in bilateral clusters involving anterior portions of the insulae and the frontal operculae as well as the right temporal pole (Supplementary Figure 2C, Supplementary Table 7). However, this difference did not reach significance in the direct statistical comparison. A conjunction analysis revealed that the pre-SMA was the only region commonly recruited by both groups (Figure $3 C$ ).

The recruitment of semantic vs. cingulo-opercular regions depends on the predictability of speech when intelligibility is increasing but not on age

Finally, we were interested in brain regions that are differentially affected by predictability when intelligibility is varied parametrically (i.e., the interaction between intelligibility and predictability). To this end, we modelled intelligibility as linear parametric modulation of high and low predictable sentences. In both age groups, we found higher activation for high vs. low predictable sentences under increasing intelligibility in several large left- and right-hemispheric clusters (Figure 4). Older participants showed increased activity in bilateral inferior parietal lobes including angular gyrus and the posterior portion of the middle and inferior temporal gyrus. Notably, the cluster in left inferior parietal cortex comprised cytoarchitectonic regions PGa, PGp and PFm, while the activation in the right hemisphere was smaller and restricted to PGp. Furthermore, several clusters were observed in subcortical and midline structures, including the precuneus, posterior cingulate cortex, the frontal pole and the accumbens (Figure 4A, left, Supplementary Table 8). Overall, the activation pattern was broadly similar in young adults, while cluster sizes were considerably larger. We observed additional activity in several subcortical regions, such as the hippocampus and the putamen in young participants. Moreover, the right-hemispheric inferior parietal cluster was not restricted to PGp but also covered PGa (Figure 4A, middle). The conjunction analysis revealed that left angular gyrus (PGa \& PGp), right angular gyrus (PGp) as well as left posterior middle temporal gyrus were recruited by both age groups (Figure $4 \mathrm{~A}$, right). Note that, opposed to the main effect of predictability, the homologous right angular gyrus seems to play a role in both age groups 
bioRxiv preprint doi: https://doi.org/10.1101/2021.12.02 470763; this version posted December 3, 2021. The copyright holder for this preprint (which was not certified by peer review) is the author/funder, who has granted bioRxiv a license to display the preprint in perpetuity. It is made available under aCC-BY-NC-ND 4.0 International license.

when sentence intelligibility is varied. There was no significant difference in activation between age groups in the direct comparison.

The opposite direction of the interaction contrast targeted brain regions sensitive to sentences of low predictability under increasing intelligibility. We found significant increases in activity in the pre-SMA and bilateral anterior insulae, extending into the frontal operculum (Figure 4B, Supplementary Table 7). These regions are associated with the domain-general cingulo-opercular network (Dosenbach et al., 2008). There was one additional cluster in the middle frontal gyrus in the old participants. Note that in contrast to the main effect of predictability reported above, older adults here indeed showed increased activity in the anterior insular regions. Young participants showed additional clusters in left and right frontal poles and left IFG. The clusters in pre-SMA and bilateral insulae were identified as common activation across both age groups and were used as seed regions for the effective connectivity analysis, together with left pMTG and bilateral AG. There were no significant differences in the group comparisons.

\section{Interaction Predictability $\mathbf{x}$ Intelligibility}

A High > low predictable, increasing intelligibility

Old adults
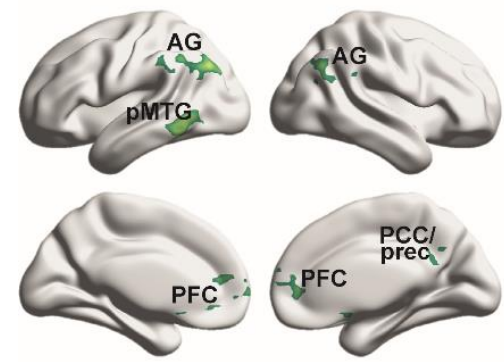

Young adults

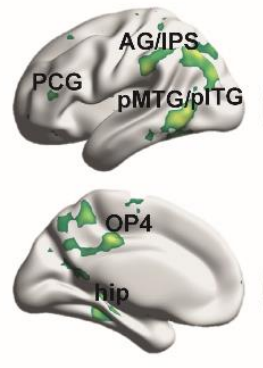

3
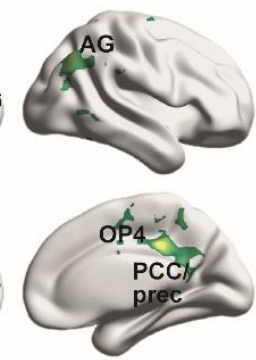

7

$\mathrm{T}$

B Low $>$ high predictable, increasing intelligibility

Old adults

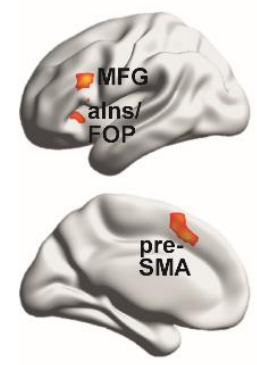

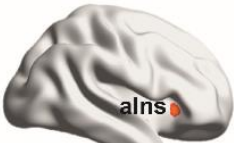

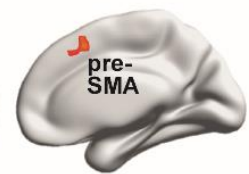

Young adults

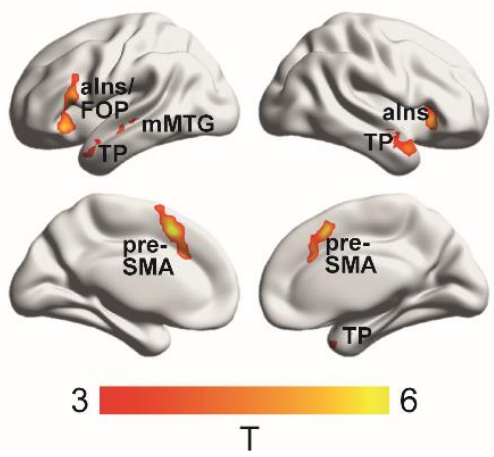

Old $\cap$ Young
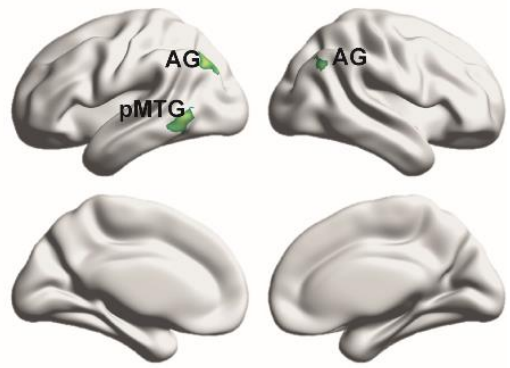

Old $\cap$ Young
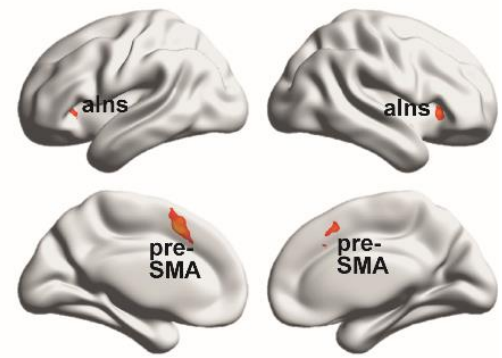

Figure 4. fMRI results showing the interaction effect of intelligibility and predictability. A Brain regions that show significant effects for the interaction contrast (high $\mathrm{x}$ increasing intelligibility $>$ low $\mathrm{x}$ increasing intelligibility), separately for old (left) and young participants (middle). The right column shows regions commonly activated by both groups. B Brain regions that show significant effects in the opposite direction of the interaction (low $\mathrm{x}$ increasing 
intelligibility $>$ high $x$ increasing intelligibility). All activation maps are thresholded at cluster-level $p_{F W E}<0.05$, with a voxel-wise threshold of $p<0.001, \mathrm{AG}=$ angular gyrus, $\mathrm{pMTG} / \mathrm{pITG}=$ posterior middle/inferior temporal gyrus, $\mathrm{PFC}=$ prefrontal cortex, $\mathrm{PCC} /$ prec $=$ posterior cingulate cortex/precuneus, $\mathrm{PCG}=$ precentral gyrus, IPS = inferior parietal sulcus, $\mathrm{OP} 4=$ parietal operculum, hip $=$ hippocampus, prec $=$ precuneus, $\mathrm{FOP}=$ frontal operculum, alns $=$ anterior insula, pre-SMA = pre-supplementary motor area, $\mathrm{TP}=$ temporal pole, $\mathrm{mMTG}=$ mid-middle temporal gyrus.

\section{High predictability modulates connectivity from semantic regions to the right anterior insula in both age groups}

At the network level, we investigated commonalities and age-related differences in effective connectivity driven by the interactive effect of predictability and intelligibility within the semantic and cingulo-opercular network and between both networks. We followed a dual-step procedure comparing (1) age-related differences in the network architecture of the cingulo-opercular network based on previous findings (Rysop et al. 2021) and (2) age-related differences in a larger network configuration containing seed regions based on the exact coordinates from the univariate conjunction analyses reported above (Figure 5A).

First, we asked whether the cingulo-opercular model that was identified as best model explaining the data of young participants would also succeed in explaining the data of older participants. Overall, the exceedance probabilities for all families of models included in the DCM analysis were remarkably low in the older subgroup, with the highest exceedance probability of 0.35 (opposed to 0.73 in young participants). No clear winning family of models could be identified. The winning model that was identified in young participants corresponded to the second probable model in the older group, with an exceedance probability of 0.24 (see Supplementary Figure 3) and an average of $14.36 \%$ explained variance (opposed to $22.13 \%$ in young participants), thus indicating that the winning model of young participants must not necessarily serve as an optimal model for older participants as well. Independent t-tests conducted on the parameter estimates that were extracted from the modulated connections of that model (i.e., winning model in young participants) indicated no significant age-related differences (see Supplementary Table 10 and Supplementary Figure 4).

As a next step, we conducted a PEB DCM analysis that is more powerful in the analysis of age-related differences in effective connectivity as it allows a larger network space encompassing seed regions from both networks of interest across both age groups. The full model yielded an average of $22.78 \%$ explained variance across both groups $(22.75 \%$ for old participants, $22.82 \%$ for young participants). We examined which connections were modulated by either high or low predictable sentences under increasing intelligibility in both groups (e.g., commonalities across participants). Here we found that high predictability had an inhibitory influence on the connection from left pMTG to right alns (Figure 5B, left; see also Table 1 and Supplementary Figure 6 for individual parameters of common modulatory effects). There was no intrinsic connection between these regions, thus these regions form a functional interaction during the task, but do not interact with each other during rest (see Supplementary 
Table 11 and 12 and Supplementary Figure 5 for intrinsic connections). Moreover, high predictability had an excitatory influence on the connection from right AG to right alns, further strengthening the positive intrinsic connection between these regions. Low predictable sentences did not have a significant effect on any of the connections, aside from weak evidence for the inhibitory influence from PMTG to right alns (posterior probability $=0.64$ ).

Young adults exhibit stronger connectivity within cingulo-opercular regions and between left insula and PMTG when sentences are highly predictable and increasingly intelligible

Next, we looked at connections that showed age-related differences in the modulatory effect of high and low predictable sentences. Age-related differences were found in the modulatory influence of high predictability on three connections: from left alns to PMTG, from left alns to pre-SMA and from pre-SMA to right alns (Figure 5B, right; see also Table 2). Coupling between these connections was stronger for younger adults. Testing connectivity values for each group against zero, revealed that the modulatory effect was negative (i.e., inhibitory) in older adults for all three connections $(p<0.05$; see Figure $5 \mathrm{C}$ for individual parameters of connections that showed age-related differences and Supplementary Figure 6 for individual parameters of common modulatory effects). In contrast, all modulatory parameters of young participants were positive (i.e., excitatory), except for the non-significant modulation of the connectivity from left anterior insula to pre-SMA $(p=0.06)$. None of the connections had a stronger coupling for older compared to younger adults. Low predictable sentences did not have a significant modulatory influence on any connection. 
A Full Model

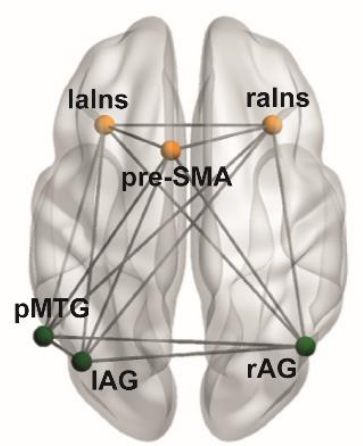

semantic seed cingulo-opercular seed

\section{C}

Participant-specific connectivity parameters

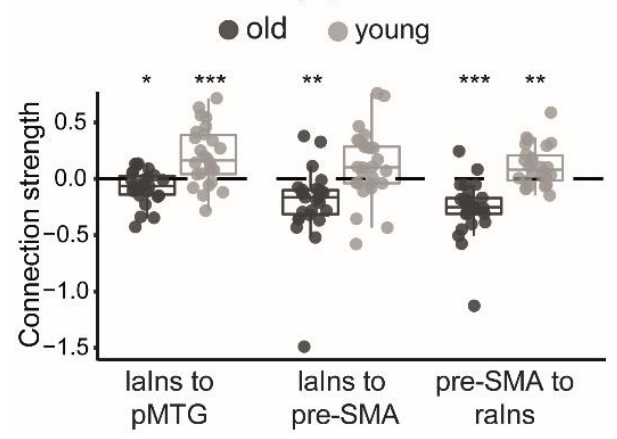

B Modulatory Parameters

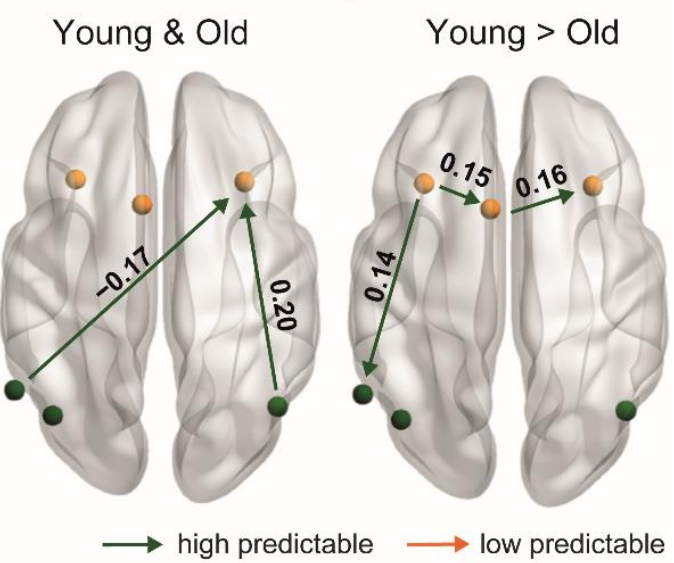

D Connectivity and behaviour (lalns to pre-SMA)

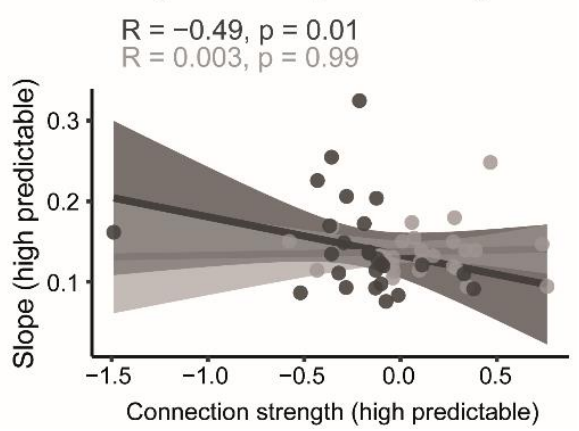

Figure 5. DCM PEB results. A Illustration of the full DCM model; semantic seeds are coloured in green, cinguloopercular seeds are coloured in orange; lalns/ralns = left/right anterior insula, pMTG = posterior middle temporal gyrus, I/rAG = left/right angular gyrus, pre-SMA = pre-supplementary motor area. B Group results of the Bayesian model average (BMA) of the modulatory parameters (B-parameters), thresholded at a posterior probability of $>$ 0.95. Modulatory effects of high (green arrow) and low (orange arrow) predictable sentences with parametrically modulated intelligibility. Left: Modulatory effects of the intelligibility x predictability interaction that are common for both groups. Right: Age-related differences (young > old adults) for the modulatory effects of high predictable sentences. C Individual modulatory parameters for high predictable sentences at each connection showing an agerelated difference. Asterisks indicate connections that significantly differ from zero, ${ }^{*}=p<0.05,{ }^{* *}=p<0.01,{ }^{* * *}=$ $p<0.001$. D Negative correlation between connectivity (modulation from left anterior insula to pre-SMA) and the slope parameter from the psychometric curve for high predictable sentences, shaded area represents $95 \%$ confidence intervals.

To test the predictive validity of the age-related connectivity differences, we performed a leave-one-out cross-validation on the three connectivity parameters that differed between age groups. The out-of-samples Pearson correlation between the predicted and actual group membership was significant for all three connections (left alns $\rightarrow$ pMTG: $r=0.29, p=0.028$; left alns $\rightarrow$ pre-SMA: $r=0.28, p=0.023$; pre-SMA $\rightarrow$ right alns: $r=0.34, p=0.006$ ). Thus, the effect sizes were large enough to predict a left-out participant's age group based on their connectivity. 
Table 1. Modulatory parameter estimates from the Bayesian model average (BMA) reflecting commonalities in connectivity across groups.

\begin{tabular}{|c|c|c|c|c|}
\hline \multicolumn{5}{|c|}{ Commonalities across age groups } \\
\hline \multirow[t]{2}{*}{ Connection } & \multicolumn{4}{|c|}{ Modulatory connectivity } \\
\hline & High * SNR & $\mathrm{Pp}$ & Low * SNR & $\mathrm{Pp}$ \\
\hline \multicolumn{5}{|c|}{ Between Network Connections } \\
\hline rAG --> ralns & 0.20 & 1 & 0.00 & 0 \\
\hline pMTG --> ralns & -0.17 & 1 & -0.09 & 0.64 \\
\hline
\end{tabular}

Table 2. Modulatory parameter estimates from the Bayesian model average (BMA) reflecting differences in connectivity between groups.

\begin{tabular}{|c|c|c|c|c|}
\hline \multicolumn{5}{|c|}{ Differences between age groups (young $>$ old) } \\
\hline \multirow[t]{2}{*}{ Connection } & \multicolumn{4}{|c|}{ Modulatory connectivity } \\
\hline & High * SNR & $\mathbf{P p}$ & Low * SNR & $\mathbf{P p}$ \\
\hline \multicolumn{5}{|c|}{ Within Cingulo-Opercular Network } \\
\hline lalns --> pre-SMA & 0.15 & 1 & 0.00 & 0.00 \\
\hline pre-SMA --> ralns & 0.16 & 1 & 0.00 & 0.00 \\
\hline
\end{tabular}

Between Network Connections

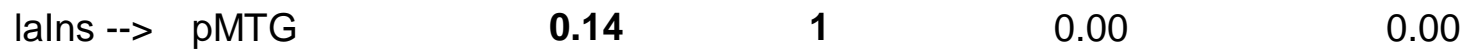

Note: Only connections with at least one parameter exceeding a posterior probability $(\mathrm{Pp})>.95$ are shown and highlighted in bold. For the differences between age groups positive values indicate stronger connectivity for young adults. See Supplementary Table 12 for differences in intrinsic connectivity.

Finally, we were interested whether age differences in connectivity strength were associated with behavioural performance. To this end, we correlated connectivity parameters that showed significant group effects with the respective parameters from the psychometric curves, representing the behavioural predictability gain (threshold and slope parameter). We found a significant negative relationship between the slope parameter of high predictable sentences (representing the sensitivity to changes in intelligibility) and the coupling strength for the connection from left anterior insula to the pre-SMA (i.e., within the cingulo-opercular network) in old participants ( $r h o=-0.49, p=0.012$; Figure 5D). Those older participants with a more negative connectivity were more sensitive to changes in intelligibility of highly predictable sentences at the behavioural level. Put differently, increased inhibition between these two domain-general regions was associated with higher accuracy at the behavioural level in old participants. This connectivity-behaviour association was not significant in young participants ( $r h o=0.046, p=0.83$ ). No other connectivity-behaviour relationships were significant. 
Together, these results demonstrate that connectivity within the semantic and the cingulo-opercular network, as well as between both networks, was mainly modulated by high predictable sentences. Younger participants exhibited stronger coupling between subregions of the cingulo-opercular network and between left anterior insula and pMTG when speech was increasingly intelligible and highly predictive compared to their older counterparts. Decreased connectivity within the cingulo-opercular network was associated with increased task performance selectively in older adults. 


\section{Discussion}

In this study, we investigated commonalities and differences during speech comprehension under challenging listening conditions in young and middle-aged to old participants. First, we found that both age groups showed a comparable behavioural predictability gain, reflected in overlapping psychometric curves. Second, depending on the semantic context of increasingly intelligible speech, both groups commonly recruited distributed left-hemispheric semantic and cingulo-opercular brain regions. Third, young and old listeners differed in effective connectivity during highly predictable and intelligible speech: Young adults exhibited overall stronger connectivity between regions of the cingulo-opercular network and between left anterior insula and pMTG. Moreover, these interactions were excitatory in young adults but inhibitory in old adults. Finally, the degree of the inhibitory influence from left anterior insula to pre-SMA was predictive of the behavioural sensitivity towards changes in intelligibility for high predictable sentences in older adults only. Our results demonstrate that the predictability gain is relatively preserved in middle-aged to older adults with good peripheral hearing when stimulus intelligibility is adjusted to individual hearing abilities. Accordingly, age-related differences at the neural level are subtle and manifest in effective connectivity, where active inhibition between cingulo-opercular regions is associated with better comprehension when speech is highly predictive.

\section{Old listeners benefit from semantic context as much as young listeners}

Our experiment was designed to assure comparable task difficulty for young and old listeners, as older listeners are known to have increased difficulties with hearing speech in challenging listening conditions even if they have relatively normal audiograms (Pichora-Fuller et al., 2017). Although we only included older participants with relatively good peripheral hearing as measured by an audiogram, they had significantly higher speech reception thresholds than young listeners. This apparent mismatch converges with the notion that hearing acuity in quiet (as measured by pure-tone audiometry) is not a good predictor of hearing abilities in noise (Schneider et al., 2010). Moreover, this stresses the importance of taking individual differences in hearing abilities into account when conducting cross-sectional experiments in the auditory domain, for instance by carefully adjusting stimulus material.

Indeed, when controlling for differences in peripheral hearing abilities, both age groups showed a comparable benefit from semantic context, suggesting that younger and older listeners did not differ in their ability to exploit contextual cues. In earlier studies, older adults showed a similar or even slightly enhanced benefit from semantic context (e.g., Pichora-Fuller et al., 1995; 1998; 2008). This was explained by a stronger recruitment of top-down contextual 
information to compensate for the age-related decline in bottom-up sensory information in older adults. If this was the case, we would have expected a relative "overuse" of semantic cues when auditory degradation was adjusted to individual hearing acuity, manifesting in a larger predictability gain in older adults. However, we did not observe age-related differences at the behavioural level. In general, our finding that older adults show a comparable beneficial effect from contextual cues is consistent with previous findings that reported a comparable predictability gain for older listeners (e.g., Humes, 1996; Goy et al., 2013; Sheldon, et al. , 2008).

\section{Age does not affect which brain regions are recruited in speech comprehension}

At the level of task-related brain activity, both age groups showed speech-sensitive engagement of left and right auditory regions, encompassing Heschl's gyrus and the superior temporal cortex, as well as the right parietal operculum. The common recruitment of bilateral primary auditory regions is well in line with the literature, where hypoactivation of the primary auditory cortex was observed in listeners with age-related hearing loss (Peelle et al., 2011) or listeners with normal hearing but worse task performance (Wong et al., 2009). With respect to age-related differences, older adults exhibited less activity in frontal regions, including right temporal pole and dorsal anterior cingulate cortex, which may reflect unspecific dedifferentiation effects related to ageing. In contrast, stronger activity in the bilateral precentral gyrus and the cerebellum for older relative to younger participants may reflect a different listening strategy such as inner rehearsal in older participants (Guediche, Holt, Laurent, Lim, \& Fiez, 2015). Indeed, a stronger contribution of the cerebellum in older adults has been previously reported during picture naming and was linked to articulatory and phonological processes (Chen \& Desmond, 2005; Ferré, Jarret, Brambati, Bellec, \& Joanette, 2020; Li et al., 2001).

With respect to the interaction of predictability and intelligibility, older participants showed broadly the same activation pattern as young adults, with generally smaller cluster sizes. Common activity across age groups was mainly found in left angular gyrus and posterior middle temporal gyrus regions associated with semantic (control) processes (Binder et al., 2009; Hoffman \& Morcom, 2018; Jackson, 2021). This is further in line with a meta-analysis (Hoffman \& Morcom, 2018) reporting overall similar activation for young and old participants in semantic tasks. In that meta-analysis, differences between age groups manifested as additional right-hemispheric activity in older adults and were observed when task performance of older participants was poor. We found no evidence for such additional domain-general activity in older adults, most likely because we assured that difficulty of the sentence repetition 
task was comparable across participants. On the contrary, young listeners showed stronger activity in an additional brain region, namely the precuneus, when contrasting high and low predictable sentences irrespective of intelligibility. Increased activity in the precuneus/posterior cingulate is often observed in semantic tasks and may serve as an interface between semantic and memory systems (Binder et al., 2009). The exact role of the precuneus/posterior cingulate complex in semantic cognition is not clear, as these regions are associated with numerous cognitive functions (see Smallwood et al., 2021; Jung \& Lambon Ralph, 2021). Age-related differences in this region might reflect a consequence of neural dedifferentiation, manifesting as a change in the relative balance of this region.

Aside from the expected association of left angular gyrus activity with the predictability gain, we also found increased activity in right angular gyrus. The effective connectivity between right angular gyrus and right anterior insula was strengthened in young and old listeners when speech was highly predictable and increasingly intelligible. These findings point towards a potential demand-related role of right angular gyrus in semantic processing. There is mounting evidence that both left and right angular gyrus are involved in (combinatorial) semantic processes (Binder et al., 2009; Golestani et al., 2013; Graessner, Zaccarella, \& Hartwigsen, 2021; Graves, Binder, Desai, Conant, \& Seidenberg, 2010; Price, Bonner, Peelle, \& Grossman, 2015). During speech comprehension under challenging listening conditions, right angular gyrus showed increased activity for related words at higher intelligibility in young participants, but at a lower threshold than left angular gyrus (Golestani et al., 2013). This supports the notion that right angular gyrus might indeed be specifically recruited during semantic processing when task demands increase.

When semantic context was low, both age groups showed increased activity in regions of the cingulo-opercular network for more intelligible speech, which was expected based on the literature (Peelle, 2018; Vaden Jr. et al., 2015, 2013, 2017) and likely helps to maintain stable performance under increased task demands (Peelle, 2018). However, contrary to our hypothesis, we did not observe a stronger recruitment of cingulo-opercular regions in older adults, which has been reported in previous work (Reuter-Lorenz \& Cappell, 2008), and was linked to successful performance in older listeners (Erb \& Obleser, 2013). The difference between the previous and present work is likely explained by our adaptive tracking paradigm which calibrated noise levels to the individual hearing abilities. Our results imply that previously reported differences between age groups may have resulted from increased task demands for older participants due to differences in peripheral hearing. 


\section{Age affects connectivity within and between networks recruited in degraded speech comprehension}

Finally, at the neural network level, we found common interaction patterns across groups but also age-related differences in effective connectivity. In both groups, task-related changes in connectivity were observed selectively for high predictable sentences (with linearly increasing intelligibility), but not for low predictable sentences. Independent of age, high predictable sentences increased the inhibitory influence of left PMTG on the right anterior insula, as well as the excitatory influence of right $A G$ on the right anterior insula. This finding points towards a complex interaction pattern of semantic-specific and domain-general areas during speech comprehension under challenging listening conditions even when task-relevant semantic information is available.

Although we did not find age-related differences at the level of activation, effective connectivity patterns within the cingulo-opercular network (left anterior insula to pre-SMA, preSMA to right anterior insula), and between cingulo-opercular and semantic regions (left anterior insula to left pMTG) differed as a function of age. Connectivity between these areas was overall stronger for young adults. Notably, the direction of the influence differed between both groups, with excitatory connectivity in young and inhibitory connectivity in old listeners. This pattern of overall reduced within-network connectivity in cognitive control networks in the ageing brain converges with several previous findings, although it remains debated whether these changes reflect processes of decline or compensation (Campbell, Grady, Ng, \& Hasher, 2012; Madden et al., 2010; O'Connell \& Basak, 2018; but see Grady et al., 2010). In our study, the degree of the inhibitory influence between cingulo-opercular regions was associated with a steeper slope of the psychometric curve for high predictable speech in older adults only. This reflects higher sensitivity towards intelligibility in highly predictable speech selectively in older adults and suggests a potential beneficial effect of inhibition within the cingulo-opercular network when semantic context can be used to predict upcoming speech. Further evidence supporting this notion comes from a recent study demonstrating that increased connectivity within domaingeneral networks was associated with less efficient behavioural performance in a semantic task in older adults (Martin, Saur, \& Hartwigsen, 2021). Importantly, the opposite pattern was observed for young adults, which converges with our finding of increased connectivity within the cingulo-opercular network in young adults. Together, the present results show that such inhibition of the interactions in the domain-general cingulo-opercular network may constitute a key mechanism underlying the predictability gain in the ageing brain.

It is noteworthy however, that younger listeners exhibited a pattern of inhibition within the cingulo-opercular network in a previous investigation (Rysop et al., 2021). In the present study, the same model appeared less suitable for older adults, as revealed by a low amount of 
explained variance captured by the model alongside low exceedance probability. This apparently conflicting finding demonstrates that effective connectivity results must be interpreted with caution and with respect to the broader context of its setup. As the PEB DCM approach yielded higher levels of explained variance, considers the (un)certainty of participants' parameters and allows a larger model space enabling the investigation of within and between network interactions, we believe that this approach is more suitable to investigate age-related differences in effective connectivity.

To our knowledge, this study represents the first investigation of age-related differences in the effective connectivity underlying the predictability gain during speech processing under challenging listening conditions. As the methodological DCM framework only allows to include a limited number of seed regions, it remains to be investigated how the presence of semantic context alters network configurations between functional networks, such as the default mode, cingulo-opercular or the fronto-parietal control network on a larger level, and how such configurations change with age.

\section{Conclusions}

The present study shows that young and old adults recruit a similar set of semantic and cingulo-opercular brain regions during speech comprehension under challenging listening conditions when intelligibility was calibrated to the individual hearing thresholds. This highlights the importance of carefully adjusting stimulus intensities to individual hearing levels in ageing research. With such precautions, age-related differences in task-related recruitment are rather subtle and manifest only at the network level. Older adults showed overall decreased connectivity between semantic and cingulo-opercular regions as well as within the cinguloopercular network. Nevertheless, the degree of inhibition within the cingulo-opercular network was positively related to speech comprehension in older adults only, demonstrating the behavioural relevance of such interactions. These findings provide new insight into age-related changes in the network configuration underlying the predictability gain. 


\section{Acknowledgements}

The authors would like to thank Isabel Gebhardt and Rebekka Luckner for assistance in data acquisition. We also thank Anke Kummer, Sylvie Neubert and Manuela Hofmann for assistance during the $\mathrm{fMRI}$ measurements. We are grateful to Julia Erb for providing the stimulus materials and to the University of Minnesota Center for Magnetic Resonance Imaging for providing the $\mathrm{fMRI}$ multiband sequence software. 
bioRxiv preprint doi: https://doi.org/10.1101/2021.12.02.470763; this version posted December 3, 2021. The copyright holder for this preprint (which was not certified by peer review) is the author/funder, who has granted bioRxiv a license to display the preprint in perpetuity. It is made available under aCC-BY-NC-ND 4.0 International license.

\section{References}

Adank, P. (2012). The neural bases of difficult speech comprehension and speech production: Two Activation Likelihood Estimation (ALE) meta-analyses. Brain and Language, 122(1), 42-54.

https://doi.org/10.1016/j.bandl.2012.04.014

Aschenbrenner, S., Tucha, O., \& Lange, K. W. (2000). Regensburger Wortflüssigkeits-Test: RWT. Hogrefe, Verlag für Psychologie.

Binder, J. R., Desai, R. H., Graves, W. W., \& Conant, L. L. (2009). Where is the semantic system? A critical review and meta-analysis of 120 functional neuroimaging studies. Cerebral Cortex, 19(12), 2767-2796. https://doi.org/10.1093/cercor/bhp055

Cameron, A. C., \& Windmeijer, F. A. G. (1997). An R-squared measure of goodness of fit for some common nonlinear regression models. Journal of Econometrics, 77(2), 329-342. https://doi.org/10.1016/S0304 4076(96)01818-0

Campbell, K. L., Grady, C. L., Ng, C., \& Hasher, L. (2012). Age differences in the frontoparietal cognitive control network: Implications for distractibility. Neuropsychologia, 50(9), 2212-2223. https://doi.org/10.1016/j.neuropsychologia.2012.05.025

Chen, S. H. A., \& Desmond, J. E. (2005). Temporal dynamics of cerebro-cerebellar network recruitment during a cognitive task. Neuropsychologia, 43(9), 1227-1237. https://doi.org/10.1016/J.NEUROPSYCHOLOGIA.2004.12.015

Darwin, C. . (2008). Listening to speech in the presence of other sounds. Philosophical Transactions of the Royal Society B: Biological Sciences, 363(1493), 1011-1021. https://doi.org/10.1098/rstb.2007.2156

Desjardins, J. L., \& Doherty, K. A. (2013). Age-Related Changes in Listening Effort for Various Types of Masker Noises. Ear \& Hearing, 34(3), 261-272. https://doi.org/10.1097/AUD.0b013e31826d0ba4

Dijkstra, N., Zeidman, P., Ondobaka, S., Van Gerven, M. A. J., \& Friston, K. (2017). Distinct Top-down and Bottom-up Brain Connectivity during Visual Perception and Imagery. Scientific Reports, 7(1), 1-9. https://doi.org/10.1038/s41598-017-05888-8

Dosenbach, N. U. F., Fair, D. A., Cohen, A. L., Schlaggar, B. L., \& Petersen, S. E. (2008). A dual-networks architecture of top-down control. Trends in Cognitive Sciences, 12(3), 99-105. https://doi.org/10.1016/j.tics.2008.01.001

Dubno, J. R., Ahlstrom, J. B., \& Horwitz, A. R. (2000). Use of context by young and aged adults with normal hearing. The Journal of the Acoustical Society of America, 107(1), 538-546. https://doi.org/10.1121/1.428322

Eckert, M. A., Teubner-Rhodes, S., \& Vaden Jr., K. I. (2016). Is listening in noise worth it? the neurobiology of speech recognition in challenging listening conditions. Ear and Hearing, 37, 101S-110S. https://doi.org/10.1097/AUD.0000000000000300

Eckert, M. A., Walczak, A., Ahlstrom, J., Denslow, S., Horwitz, A., \& Dubno, J. R. (2008). Age-related effects on word recognition: Reliance on cognitive control systems with structural declines in speech-responsive cortex. JARO - Journal of the Association for Research in Otolaryngology, 9(2), 252-259. https://doi.org/10.1007/s10162-008-0113-3

Eickhoff, S. B., Paus, T., Caspers, S., Grosbras, M.-H., Evans, A. C., Zilles, K., \& Amunts, K. (2007). Assignment of functional activations to probabilistic cytoarchitectonic areas revisited. Neurolmage, 36(3), 511-521. https://doi.org/10.1016/j.neuroimage.2007.03.060

Eickhoff, S. B., Stephan, K. E., Mohlberg, H., Grefkes, C., Fink, G. R., Amunts, K., \& Zilles, K. (2005). A new SPM toolbox for combining probabilistic cytoarchitectonic maps and functional imaging data. Neurolmage, 25(4), 1325-1335. https://doi.org/10.1016/j.neuroimage.2004.12.034

Erb, J., Henry, M. J., Eisner, F., \& Obleser, J. (2012). Auditory skills and brain morphology predict individual differences in adaptation to degraded speech. Neuropsychologia, 50(9), 2154-2164. https://doi.org/10.1016/j.neuropsychologia.2012.05.013

Erb, J., \& Obleser, J. (2013). Upregulation of cognitive control networks in older adults' speech comprehension. Frontiers in Systems Neuroscience, 7(December), 1-13. https://doi.org/10.3389/fnsys.2013.00116

Federmeier, K. D. (2007). Thinking ahead: The role and roots of prediction in language comprehension. Psychophysiology, 44(4), 491-505. https://doi.org/10.1111/j.1469-8986.2007.00531.x 
bioRxiv preprint doi: https://doi.org/10.1101/2021.12.02.470763; this version posted December 3, 2021. The copyright holder for this preprint (which was not certified by peer review) is the author/funder, who has granted bioRxiv a license to display the preprint in perpetuity. It is made available under aCC-BY-NC-ND 4.0 International license.

Ferré, P., Jarret, J., Brambati, S. M., Bellec, P., \& Joanette, Y. (2020). Task-Induced Functional Connectivity of Picture Naming in Healthy Aging: The Impacts of Age and Task Complexity. Neurobiology of Language, 1(2), 161-184. https://doi.org/10.1162/nol_a_00007

Fitzhugh, M. C., Braden, B. B., Sabbagh, M. N., Rogalsky, C., \& Baxter, L. C. (2019). Age-Related Atrophy and Compensatory Neural Networks in Reading Comprehension. Journal of the International Neuropsychological Society, 25(6), 569-582. https://doi.org/10.1017/S1355617719000274

Fitzhugh, M. C., Schaefer, S. Y., Baxter, L. C., \& Rogalsky, C. (2021). Cognitive and neural predictors of speech comprehension in noisy backgrounds in older adults. Language, Cognition and Neuroscience, 36(3), 269287. https://doi.org/10.1080/23273798.2020.1828946

Folstein, M. F., Folstein, S. E., \& McHugh, P. R. (1975). "Mini-mental state." Journal of Psychiatric Research, 12(3), 189-198. https://doi.org/10.1016/0022-3956(75)90026-6

Friston, K. J., Harrison, L., \& Penny, W. (2003). Dynamic causal modelling. Neurolmage, 19(4), 1273-1302. https://doi.org/10.1016/S1053-8119(03)00202-7

Friston, K. J., Litvak, V., Oswal, A., Razi, A., Stephan, K. E., van Wijk, B. C. M., ... Zeidman, P. (2016). Bayesian model reduction and empirical Bayes for group (DCM) studies. Neurolmage, 128, 413-431. https://doi.org/10.1016/j.neuroimage.2015.11.015

Friston, K. J., Worsley, K. J., Frackowiak, R. S. J., Mazziotta, J. C., \& Evans, A. C. (1994). Assessing the significance of focal activations using their spatial extent. Human Brain Mapping, 1(3), 210-220. https://doi.org/10.1002/hbm.460010306

Fründ, I., Haenel, N. V., \& Wichmann, F. A. (2011). Inference for psychometric functions in the presence of nonstationary behavior. Journal of Vision, 11(6), 1-19. https://doi.org/10.1167/11.6.16

Golestani, N., Hervais-Adelman, A., Obleser, J., \& Scott, S. K. (2013). Semantic versus perceptual interactions in neural processing of speech-in-noise. Neurolmage, 79, 52-61. https://doi.org/10.1016/j.neuroimage.2013.04.049

Gordon-Salant, S. (2005). Hearing loss and aging: New research findings and clinical implications. Journal of Rehabilitation Research and Development, 42(4 SUPPL. 2), 9-24. https://doi.org/10.1682/JRRD.2005.01.0006

Gordon-Salant, S., \& Fitzgibbons, P. J. (1995). Recognition of Multiply Degraded Speech by Young and Elderly Listeners. Journal of Speech, Language, and Hearing Research, 38(5), 1150-1156. https://doi.org/10.1044/jshr.3805.1150

Goy, H., Pelletier, M., Coletta, M., \& Pichora- Fuller, M. K. (2013). The Effects of Semantic Context and the Type and Amount of Acoustic Distortion on Lexical Decision by Younger and Older Adults. Journal of Speech, Language, and Hearing Research, 56(6), 1715-1732. https://doi.org/10.1044/1092-4388(2013/12-0053)

Grady, C. L., Protzner, A. B., Kovacevic, N., Strother, S. C., Afshin-Pour, B., Wojtowicz, M., ... Mclntosh, A. R. (2010). A Multivariate Analysis of Age-Related Differences in Default Mode and Task-Positive Networks across Multiple Cognitive Domains. Cerebral Cortex, 20(6), 1432-1447. https://doi.org/10.1093/cercor/bhp207

Graessner, A., Zaccarella, E., \& Hartwigsen, G. (2021). Differential contributions of left-hemispheric language regions to basic semantic composition. Brain Structure and Function, 226(2), 501-518. https://doi.org/10.1007/s00429-020-02196-2

Graves, W. W., Binder, J. R., Desai, R. H., Conant, L. L., \& Seidenberg, M. S. (2010). Neural correlates of implicit and explicit combinatorial semantic processing. Neurolmage, 53(2), 638-646. https://doi.org/10.1016/j.neuroimage.2010.06.055

Guediche, S., Holt, L. L., Laurent, P., Lim, S., \& Fiez, J. A. (2015). Evidence for Cerebellar Contributions to Adaptive Plasticity in Speech Perception. Cerebral Cortex, 25(July), 1867-1877. https://doi.org/10.1093/cercor/bht428

Halai, A. D., Welbourne, S. R., Embleton, K., \& Parkes, L. M. (2014). A comparison of dual gradient-echo and spin-echo fMRI of the inferior temporal lobe. Human Brain Mapping, 35(8), 4118-4128. https://doi.org/10.1002/hbm.22463

Hartwigsen, G., Golombek, T., \& Obleser, J. (2015). Repetitive transcranial magnetic stimulation over left angular gyrus modulates the predictability gain in degraded speech comprehension. Cortex, 68, 100-110. https://doi.org/10.1016/j.cortex.2014.08.027

Hoffman, P., \& Morcom, A. M. (2018). Age-related changes in the neural networks supporting semantic cognition: A meta-analysis of 47 functional neuroimaging studies. Neuroscience and Biobehavioral Reviews, 
bioRxiv preprint doi: https://doi.org/10.1101/2021.12.02.470763; this version posted December 3, 2021. The copyright holder for this preprint (which was not certified by peer review) is the author/funder, who has granted bioRxiv a license to display the preprint in perpetuity. It is made available under aCC-BY-NC-ND 4.0 International license.

84(November 2017), 134-150. https://doi.org/10.1016/j.neubiorev.2017.11.010

Humes, L. E. (1996). Speech understanding in the elderly. Journal of American Audiology, 7(3), 161-167.

Jackson, R. L. (2021). The neural correlates of semantic control revisited. Neurolmage, 224(September 2020), 117444. https://doi.org/10.1016/j.neuroimage.2020.117444

Jefferies, E. (2013). The neural basis of semantic cognition: Converging evidence from neuropsychology , neuroimaging and TMS. Cortex, 49(3), 611-625. https://doi.org/10.1016/j.cortex.2012.10.008

Jung, J., \& Lambon Ralph, M. A. (2021). Distinct but cooperating brain networks supporting semantic cognition. BioRxiv, (July). https://doi.org/10.1101/2021.07.19.452716

Kalikow, D. N., Stevens, K. N., \& Elliott, L. L. (1977). Development of a test of speech intelligibility in noise using sentence materials with controlled word predictability. The Journal of the Acoustical Society of America, 61(5), 1337-1351. https://doi.org/10.1121/1.381436

Kass, R. E., \& Raftery, A. E. (1995). Bayes Factors. Journal of the American Statistical Association, 90(430), 773-795. https://doi.org/10.1080/01621459.1995.10476572

Li, S.-C., Lindenberger, U., \& Sikström, S. (2001). Aging cognition: from neuromodulation to representation. Trends in Cognitive Sciences, 5(11), 479-486. https://doi.org/10.1016/S1364-6613(00)01769-1

Madden, D. J., Costello, M. C., Dennis, N. A., Davis, S. W., Shepler, A. M., Spaniol, J., ... Cabeza, R. (2010). Adult age differences in functional connectivity during executive control. Neurolmage, 52(2), 643-657. https://doi.org/10.1016/j.neuroimage.2010.04.249

Marreiros, A. C., Kiebel, S. J., \& Friston, K. J. (2008). Dynamic causal modelling for fMRI: A two-state model. Neurolmage, 39(1), 269-278. https://doi.org/10.1016/j.neuroimage.2007.08.019

Martin, S., Saur, D., \& Hartwigsen, G. (2021). Age-dependent contribution of domain-general networks to semantic cognition. Cerebral Cortex, 2020.11.06.371153. Retrieved from https://doi.org/10.1101/2020.11.06.371153

Miller, G. A. (1947). The masking of speech. Psychological Bulletin, 44(2), 105-129. https://doi.org/10.1037/h0055960

Nichols, T., Brett, M., Andersson, J., Wager, T., \& Poline, J.-B. (2005). Valid conjunction inference with the minimum statistic. Neurolmage, 25(3), 653-660. https://doi.org/10.1016/j.neuroimage.2004.12.005

O'Connell, M. A., \& Basak, C. (2018). Effects of task complexity and age-differences on task-related functional connectivity of attentional networks. Neuropsychologia, 114(August 2017), 50-64. https://doi.org/10.1016/j.neuropsychologia.2018.04.013

Obleser, J., \& Kotz, S. A. (2010). Expectancy constraints in degraded speech modulate the language comprehension network. Cerebral Cortex, 20(3), 633-640. https://doi.org/10.1093/cercor/bhp128

Obleser, J., Wise, R. J. S., Dresner, A. M., \& Scott, S. K. (2007). Functional Integration across Brain Regions Improves Speech Perception under Adverse Listening Conditions. Journal of Neuroscience, 27(9), 22832289. https://doi.org/10.1523/JNEUROSCI.4663-06.2007

Oldfield, R. C. (1971). The assessment of handedness: The Edinburgh inventory. Neuropsychologia.

Payne, B. R., \& Federmeier, K. D. (2018). Contextual constraints on lexico-semantic processing in aging: Evidence from single-word event-related brain potentials. Brain Research, 1687, 117-128. https://doi.org/10.1016/j.brainres.2018.02.021

Peelle, J. E. (2012). The hemispheric lateralization of speech processing depends on what "speech" is: a hierarchical perspective. Frontiers in Human Neuroscience, 6(November), 1-4. https://doi.org/10.3389/fnhum.2012.00309

Peelle, J. E. (2018). Listening Effort: How the Cognitive Consequences of Acoustic Challenge Are Reflected in Brain and Behavior. Ear and Hearing, 39(2), 204-214. https://doi.org/10.1097/AUD.0000000000000494

Peelle, J. E., Troiani, V., Grossman, M., \& Wingfield, A. (2011). Hearing Loss in Older Adults Affects Neural Systems Supporting Speech Comprehension. The Journal of Neuroscience, 31(35), 12638-12643. https://doi.org/10.1523/JNEUROSCI.2559-11.2011

Penny, W., Stephan, K. E., Daunizeau, J., Rosa, M. J., Friston, K. J., Schofield, T. M., \& Leff, A. P. (2010). Comparing families of dynamic causal models. PLoS Computational Biology, 6(3). https://doi.org/10.1371/journal.pcbi.1000709

Pichora-Fuller, M. K. (2008). Use of supportive context by younger and older adult listeners: Balancing bottom-up 
bioRxiv preprint doi: https://doi.org/10.1101/2021.12.02.470763; this version posted December 3, 2021. The copyright holder for this preprint (which was not certified by peer review) is the author/funder, who has granted bioRxiv a license to display the preprint in perpetuity. It is made available under aCC-BY-NC-ND 4.0 International license.

and top-down information processing. International Journal of Audiology, 47(SUPPL. 2). https://doi.org/10.1080/14992020802307404

Pichora-Fuller, M. K., Alain, C., \& Schneider, B. A. (2017). Older Adults at the Cocktail Party. In J. C. Middlebrooks, J. Z. . Simon, A. N. . Popper, \& R. R. Fay (Eds.), The Auditory System at the Cocktail Party (1st ed., pp. 227-259). Springer. https://doi.org/10.1007/978-3-319-51662-2_9

Pichora-Fuller, M. K., Schneider, B. A., \& Daneman, M. (1995). How young and old adults listen to and remember speech in noise. The Journal of the Acoustical Society of America, 97(1), 593-608. https://doi.org/10.1121/1.412282

Price, A. R., Bonner, M. F., Peelle, J. E., \& Grossman, M. (2015). Converging evidence for the neuroanatomic basis of combinatorial semantics in the angular gyrus. Journal of Neuroscience, 35(7), 3276-3284. https://doi.org/10.1523/JNEUROSCI.3446-14.2015

Reitan, R. M. (1958). Validity of the Trail Making Test as an Indicator of Organic Brain Damage. Perceptual and Motor Skills, 8(3), 271-276. https://doi.org/10.2466/pms.1958.8.3.271

Reuter-Lorenz, P. A., \& Cappell, K. A. (2008). Neurocognitive Aging and the Compensation Hypothesis. Current Directions in Psychological Science, 17(3), 177-182. https://doi.org/10.1111/j.1467-8721.2008.00570.x

Rysop, A. U., Schmitt, L. M., Obleser, J., \& Hartwigsen, G. (2021). Neural modelling of the semantic predictability gain under challenging listening conditions. Human Brain Mapping, 42(1), 110-127. https://doi.org/10.1002/hbm.25208

Salthouse, T. A., Atkinson, T. M., \& Berish, D. E. (2003). Executive Functioning as a Potential Mediator of AgeRelated Cognitive Decline in Normal Adults. Journal of Experimental Psychology: General, 132(4), 566594. https://doi.org/10.1037/0096-3445.132.4.566

Schneider, B. A., Pichora-Fuller, M. K., \& Daneman, M. (2010). The effects of senescant changes in audition and cognition on spoken languge comprehension. In S. Gordon-Salant, R. D. Frisina, A. N. Popper, \& R. R. Fay (Eds.), Springer Handbook of Auditory Research: The Aging Auditory System: Perceptual Characterizsation and Neural Bases of Presbycusis (pp. 167-210).

Seghier, M. L. (2013). The Angular Gyrus. The Neuroscientist, 19(1), 43-61. https://doi.org/10.1177/1073858412440596

Shannon, R. V., Zeng, F.-G., Kamath, V., Wygonski, J., \& Ekelid, M. (1995). Speech Recognition with Primarily Temporal Cues. Science, 270(5234), 303-304. https://doi.org/10.1126/science.270.5234.303

Sheldon, S., Pichora-Fuller, M. K., \& Schneider, B. A. (2008). Priming and sentence context support listening to noise-vocoded speech by younger and older adults. The Journal of the Acoustical Society of America, 123(1), 489-499. https://doi.org/10.1121/1.2783762

Siegel, J. S., Power, J. D., Dubis, J. W., Vogel, A. C., Church, J. A., Schlaggar, B. L., \& Petersen, S. E. (2014). Statistical improvements in functional magnetic resonance imaging analyses produced by censoring highmotion data points. Human Brain Mapping, 35(5), 1981-1996. https://doi.org/10.1002/hbm.22307

Smallwood, J., Bernhardt, B. C., Leech, R., Bzdok, D., Jefferies, E., \& Margulies, D. S. (2021). The default mode network in cognition: a topographical perspective. Nature Reviews Neuroscience, 22(8), 503-513. https://doi.org/10.1038/s41583-021-00474-4

Stephan, K. E., Penny, W., Daunizeau, J., Moran, R. J., \& Friston, K. J. (2009). Bayesian model selection for group studies. Neurolmage, 46(4), 1004-1017. https://doi.org/10.1016/j.neuroimage.2009.03.025

Uddin, L. Q., Yeo, B. T. T., \& Spreng, R. N. (2019). Towards a Universal Taxonomy of Macro-scale Functional Human Brain Networks. Brain Topography, (0123456789). https://doi.org/10.1007/s10548-019-00744-6

Vaden Jr., K. I., Kuchinsky, S. E., Ahlstrom, J., Dubno, J. R., \& Eckert, M. A. (2015). Cortical Activity Predicts Which Older Adults Recognize Speech in Noise and When. Journal of Neuroscience, 35(9), 3929-3937. https://doi.org/10.1523/JNEUROSCI.2908-14.2015

Vaden Jr., K. I., Kuchinsky, S. E., Cute, S. L., Ahlstrom, J., Dubno, J. R., \& Eckert, M. A. (2013). The CinguloOpercular Network Provides Word-Recognition Benefit. Journal of Neuroscience, 33(48), 18979-18986. https://doi.org/10.1523/JNEUROSCI.1417-13.2013

Vaden Jr., K. I., Teubner-Rhodes, S., Ahlstrom, J., Dubno, J. R., \& Eckert, M. A. (2017). Cingulo-opercular activity affects incidental memory encoding for speech in noise. Neurolmage, 157(June), 381-387. https://doi.org/10.1016/j.neuroimage.2017.06.028

Verhaeghen, P. (2003). Aging and vocabulary score: A meta-analysis. Psychology and Aging, 18(2), 332-339. https://doi.org/10.1037/0882-7974.18.2.332 
bioRxiv preprint doi: https://doi.org/10.1101/2021.12.02.470763; this version posted December 3, 2021. The copyright holder for this preprint (which was not certified by peer review) is the author/funder, who has granted bioRxiv a license to display the preprint in perpetuity. It is made available under aCC-BY-NC-ND 4.0 International license.

Wagenmakers, E.-J., Love, J., Marsman, M., Jamil, T., Ly, A., Verhagen, J., ... Morey, R. D. (2018). Bayesian inference for psychology. Part II: Example applications with JASP. Psychonomic Bulletin \& Review, 25(1), 58-76. https://doi.org/10.3758/s13423-017-1323-7

Wechsler, D. (1955). Manual for the Wechsler Adult Intelligence Scale. Manual for the Wechsler Adult Intelligence Scale. Oxford, England: Psychological Corp.

Wilsch, A., Henry, M. J., Herrmann, B., Maess, B., \& Obleser, J. (2015). Alpha Oscillatory Dynamics Index Temporal Expectation Benefits in Working Memory. Cerebral Cortex, 25(7), 1938-1946. https://doi.org/10.1093/cercor/bhu004

Wingfield, A., Amichetti, N. M., \& Lash, A. (2015). Cognitive aging and hearing acuity: Modeling spoken language comprehension. Frontiers in Psychology, 6(MAY), 1-13. https://doi.org/10.3389/fpsyg.2015.00684

Wingfield, A., \& Stine-Morrow, E. A. L. (2000). Language and Speech. In F. I. M. Craik \& T. A. Salthouse (Eds.), The handbook of aging and cognition (pp. 359-416).

Wlotko, E. W., Federmeier, K. D., \& Kutas, M. (2012). To predict or not to predict: Age-related differences in the use of sentential context. Psychology and Aging, 27(4), 975-988. https://doi.org/10.1037/a0029206

Wong, P. C. M., Jin, J. X., Gunasekera, G. M., Abel, R., Lee, E. R., \& Dhar, S. (2009). Aging and cortical mechanisms of speech perception in noise. Neuropsychologia, 47(3), 693-703. https://doi.org/10.1016/j.neuropsychologia.2008.11.032

Xia, M., Wang, J., \& He, Y. (2013). BrainNet Viewer: A Network Visualization Tool for Human Brain Connectomics. PLoS ONE, 8(7), e68910. https://doi.org/10.1371/journal.pone.0068910

Zeidman, P., Jafarian, A., Corbin, N., Seghier, M. L., Razi, A., Price, C. J., \& Friston, K. J. (2019). A guide to group effective connectivity analysis, part 1: First level analysis with DCM for fMRI. Neurolmage, 200(June), 174-190. https://doi.org/10.1016/j.neuroimage.2019.06.031

Zeidman, P., Jafarian, A., Seghier, M. L., Litvak, V., Cagnan, H., Price, C. J., \& Friston, K. J. (2019). A guide to group effective connectivity analysis, part 2: Second level analysis with PEB. Neurolmage, 200(June), 1225. https://doi.org/10.1016/j.neuroimage.2019.06.032 